\title{
Observation of Three-Photon Spontaneous Parametric Down-Conversion in a Superconducting Parametric Cavity
}

\author{
C. W. Sandbo Chang, ${ }^{1}$ Carlos Sabín, ${ }^{2}$ P. Forn-Díaz@ ${ }^{3,4}$ Fernando Quijandría $\odot,{ }^{5}$ A. M. Vadiraj $\odot,{ }^{1}$ \\ I. Nsanzineza, ${ }^{1}$ G. Johansson, ${ }^{5}$ and C. M. Wilson $\circledast^{1, *}$ \\ ${ }^{1}$ Institute for Quantum Computing and Electrical and Computer Engineering, University of Waterloo, \\ Waterloo, Ontario N2L 3G1, Canada \\ ${ }^{2}$ Instituto de Física Fundamental, CSIC, Serrano, 113-bis, 28006 Madrid, Spain \\ ${ }^{3}$ Institut de Física d'Altes Energies (IFAE), The Barcelona Institute of Science and Technology (BIST), \\ Bellaterra (Barcelona) 08193, Spain \\ ${ }^{4}$ Barcelona Supercomputing Center-CNS, Barcelona 08034, Spain \\ ${ }^{5}$ Microtechnology and Nanoscience, MC2, Chalmers University of Technology, \\ SE-412 96 Göteborg, Sweden
}

(Received 17 July 2019; revised manuscript received 17 October 2019; published 16 January 2020)

\begin{abstract}
Spontaneous parametric down-conversion (SPDC) has been a key enabling technology in exploring quantum phenomena and their applications for decades. For instance, traditional SPDC, which splits a high-energy pump photon into two lower-energy photons, is a common way to produce entangled photon pairs. Since the early realizations of SPDC, researchers have thought to generalize it to higher order, e.g., to produce entangled photon triplets. However, directly generating photon triplets through a single SPDC process has remained elusive. Here, using a flux-pumped superconducting parametric cavity, we demonstrate direct three-photon SPDC, with photon triplets generated in a single cavity mode or split between multiple modes. With strong pumping, the states can be quite bright, with flux densities exceeding 60 photons per second per hertz. The observed states are strongly non-Gaussian, which has important implications for potential applications. In the single-mode case, we observe a triangular star-shaped distribution of quadrature voltages, indicative of the long-predicted "star state." The observed state shows strong thirdorder correlations, as expected for a state generated by a cubic Hamiltonian. By pumping at the sum frequency of multiple modes, we observe strong three-body correlations between multiple modes, strikingly, in the absence of second-order correlations. We further analyze the third-order correlations under mode transformations by the symplectic symmetry group, showing that the observed transformation properties serve to "fingerprint" the specific cubic Hamiltonian that generates them. The observed non-Gaussian, thirdorder correlations represent an important step forward in quantum optics and may have a strong impact on quantum communication with microwave fields as well as continuous-variable quantum computation.
\end{abstract}

DOI: 10.1103/PhysRevX.10.011011

Subject Areas: Quantum Physics,

Quantum Information,

Superconductivity

\section{INTRODUCTION}

For over 30 years, spontaneous parametric down-conversion (SPDC) has been a workhorse for quantum optics. Famously known as a process which generates photons in pairs from a single pump, it has had a crucial role in fundamental tests of quantum theory [1-3] as well as many

\footnotetext{
* Corresponding author. chris.wilson@uwaterloo.ca

Published by the American Physical Society under the terms of the Creative Commons Attribution 4.0 International license. Further distribution of this work must maintain attribution to the author(s) and the published article's title, journal citation, and DOI.
}

applications in quantum information processing [4-6]. Spanning frequencies from optical to microwave, SPDC has a central role, for instance, in quantum-limited amplifiers [7] and sources of nonclassical light, including squeezed states [8,9], Fock states [10], and entangled photon pairs [11]. This broad and important set of phenomena has been referred to as "two-photon quantum optics" $[12,13]$. From early days, generalizations of the standard two-photon SPDC have been explored, but this endeavor has proven difficult both theoretically [14-23] and experimentally [24,25].

Even the pursuit of the next-order, three-photon SPDC which would create photon triplets, has gone on for decades [26-29]. This process has been studied theoretically in the context of generalized squeezing $[14,16,30]$, where the 
quantum vacuum is shaped by the action of nonquadratic Hamiltonians [31]. The non-Gaussian nature of these higher-order squeezed states could make them a resource for universal quantum computation with linear optics [32-35]. In particular, cubic squeezing is one path to generate the "magic" cubic-phase state of Gottesman, Kitaev, and Preskill [36] that enables error correction in continuous-variable quantum computing. Three-photon SPDC has also been studied as a source of more sophisticated, three-photon entangled states, such as GreenbergerHorne-Zeilinger states [37], as well as heralded entangled pairs [38]. These states would be useful, e.g., for novel quantum communication protocols such as quantum secret sharing [39]. Despite the great potential of multiphoton down-conversion and generalized squeezing, their experimental demonstration has remained elusive. The majority of the experimental work has been done in the optical frequency domain, where the relatively weak nonlinearities of optical materials present significant experimental challenges. It has more recently been realized that the strong nonlinearity of Josephson junctions facilitates a range of experiments in the microwave domain that are very challenging in the optical domain.

Following this theme, we report an experimental implementation of three-photon SPDC, in the microwave domain, producing generalized squeezed states, in particular, trisqueezed states. This implementation is done using a flux-pumped, superconducting parametric resonator. By the choice of pump frequency, we can alternately produce degenerate three-photon down-conversion to a single mode or nondegenerate three-photon down-conversion to three distinct modes. Furthermore, we can produce a hybrid version to two modes, where two photons are degenerate and one is nondegenerate. Our triplet source is bright, producing a propagating photon flux with a flux density controllable from less than 1 to greater than 60 photons per second per hertz over a bandwidth of hundreds of kilohertz, comparable to ordinary two-photon down-conversion (TPDC) experiments $[40,41]$. The high flux allows us to perform a detailed analysis of the novel phase-space distributions and strongly non-Gaussian statistics of the states. For instance, we clearly see strong three-body correlations in the absence of normal two-body correlations (covariance). The symmetry properties of these correlations allow us to "fingerprint" the Hamiltonians that created them, clearly demonstrating that states are generated by a family of pure cubic Hamiltonians with little contamination from typical quadratic processes. These results form the basis of an exciting new paradigm of three-photon quantum optics.

\section{BACKGROUND}

Since the first applications of SPDC were realized with a quadratic Hamiltonian, generalizing squeezing with higher-order Hamiltonians has been a topic of intense research $[14,16,18]$. In the case of single-mode SPDC, a generalized squeezing process can be described by the $k$ th-order SPDC Hamiltonian

$$
\hat{H}=\hbar g_{k}\left(\alpha^{*} \hat{a}^{k}+\alpha \hat{a}^{\dagger k}\right),
$$

where $\alpha$ represents the pump (under the parametric approximation), $g_{k}$ is the $k$ th-order coupling constant, and $\hat{a}$ is the annihilation operator of the single mode. First discussed in the literature in the mid-1980s, early work [14] concluded that generalized squeezing processes were unphysical. Subsequent work [16] showed that the issues were mainly mathematical and calculated the first phase-space distributions of generalized states using novel analytical continuation techniques. It was also later shown that the apparent divergences that appeared under the parametric approximation were removed when the pump was quantized [18]. Despite the early theoretical challenges, continued research was motivated by the novel nonGaussian statistics and nonclassical properties, including Wigner negativity [19], absent in conventional squeezed states but predicted in the higher-order squeezed states. These many-photon states continue to be attractive and potentially useful in various quantum information processing applications.

Meanwhile, the experimental implementation of an elementary SPDC process capable of generating three or more photons has remained an outstanding challenge [42]. In the optical domain, experiments using cascaded SPDC and photon generation by a quantum dot have successfully generated three-photon correlations $[37,38,43]$. However, the triplet generation rate in these schemes is small, making a detailed analysis of the three-photon states or the underlying Hamiltonian difficult. These schemes also lack explicit control over the down-converted frequencies, which make it difficult, for instance, to generate singlemode photon triplets. In the microwave domain, classical period-tripling parametric oscillations have recently been demonstrated with a parametric superconducting cavity, with a third-subharmonic oscillation observed when a strong flux pump is applied [44]. There have also been recent theoretical studies of quantum effects in periodtripled parametric oscillators [45].

Superconducting parametric cavities and resonators, based on superconducting quantum interference devices (SQUIDs), are an important device family widely used for applications requiring TPDC $[40,46,47]$. In these devices, leading-order terms of the full cosine nonlinearity of the SQUID are used to generate two-photon down-conversion. As described below in more detail, for this work, we modify and extend these devices to access higher-order nonlinearities of the SQUID.

Our device is a quarter-wavelength coplanar waveguide resonator terminated by a SQUID at one end, with the other end capacitively overcoupled $(Q \approx 7000)$ to a nominally 
$Z_{0}=50 \Omega$ line. The fundamental mode has a relatively low frequency of around $1 \mathrm{GHz}$ such that there are three higher-order modes accessible within our 4-8 GHz measurement bandwidth. Impedance engineering $[41,48,49]$ is used to make the mode spacing nondegenerate, yielding mode frequencies of approximately 4, 6, and $7 \mathrm{GHz}$. Parametric processes are driven by a microwave pump inductively coupled to the SQUID, modulating the boundary condition of the cavity. By exploiting kinetic inductance (Fig. 1) and other improvements, we increase the pump coupling by roughly $30 \mathrm{~dB}$ compared to previous designs [49], a crucial element in realizing higher-order parametric processes.

Many of the parametric cavities and resonators described above employ a nominally symmetric SQUID, where the two Josephson junctions that comprise the SQUID are designed to have the same Josephson energy. This symmetry comes with various advantages such as maximizing the frequency tunability of the cavities. However, it also suppresses cubic interactions between the cavity modes due to the even symmetry of the pure cosine nonlinearity of the symmetric SQUID. To access cubic (odd) nonlinearities, we must therefore break the symmetry of the SQUID. To understand this requirement in more detail, we can write the

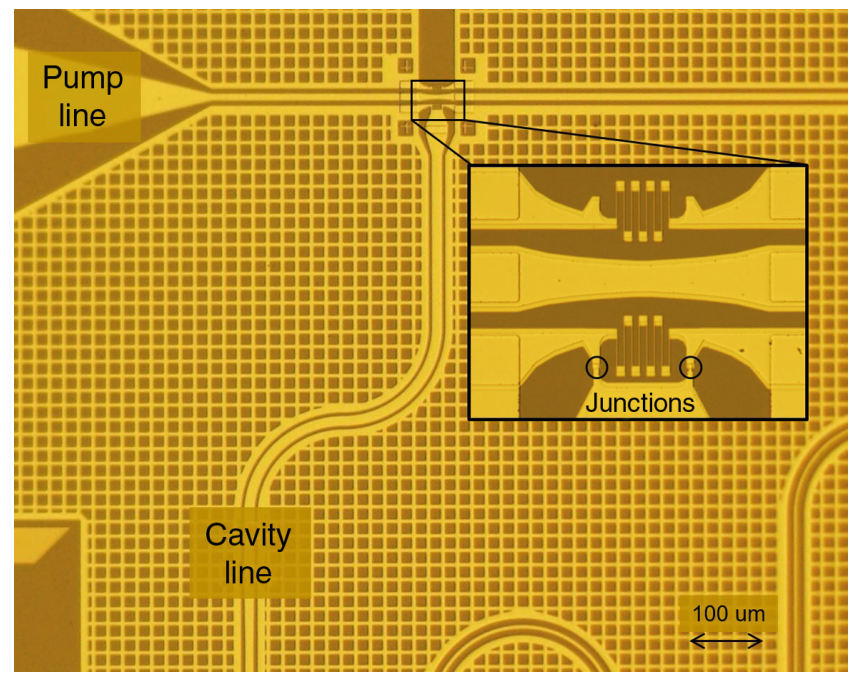

FIG. 1. Meandered SQUID design with improved pump coupling. The narrow, meandered path is shared between the SQUID and the ground plane of the pump line. The kinetic inductance of the meander then directly couples the pump current in the ground plane to the SQUID phase. The center conductor of the pump line is narrowed to reduce its magnetic coupling to the SQUID, which contributes with the opposite sign of the kinetic coupling. Following microwave simulations, a mirrored structure is patterned in the top ground plane, such that the ground current divides evenly between the two ground planes. Compared to previous designs, we find a reduction in the pump strength required for TPDC by 3 orders of magnitude. The reduction greatly reduces the spurious effects of strong pumping and is critical to realizing three-photon down-conversion. general sinusoidal Hamiltonian of an asymmetric SQUID, coupling the cavity flux $\hat{\Phi}_{\text {cav }}$ to the external pump flux $\hat{\Phi}_{\text {ext }}$ as

$$
\begin{aligned}
\hat{H}_{\mathrm{SQ}} & =E_{J}\left(\hat{\Phi}_{\mathrm{ext}}\right) \cos \left(2 \pi \hat{\Phi}_{\mathrm{cav}} / \Phi_{0}-\alpha\right), \\
E_{J}\left(\hat{\Phi}_{\mathrm{ext}}\right) & =\sqrt{E_{J, 1}^{2}+E_{J, 2}^{2}+2 E_{J, 1}^{2} E_{J, 2}^{2} \cos \left(2 \pi \frac{\hat{\Phi}_{\mathrm{ext}}}{\Phi_{0}}\right)}, \\
\alpha & =\arctan \left[\tan \left(\pi \frac{\hat{\Phi}_{\mathrm{ext}}}{\Phi_{0}}\right) \frac{E_{J, 1}-E_{J, 2}}{E_{J, 1}+E_{J, 2}}\right] .
\end{aligned}
$$

$E_{J}\left(\hat{\Phi}_{\text {ext }}\right)$ is the flux-dependent Josephson energy of the SQUID [Eq. (A5)]. $E_{J}$ is tuned by the external flux $\hat{\Phi}_{\text {ext }}=\hat{\Phi}_{p}+\Phi_{\text {bias }}$, composed of the ac pump flux and dc flux bias. $E_{J, i}$ are the Josephson energies of the individual junctions. $\alpha$ is the effective cavity flux bias arising from the SQUID asymmetry, which is necessary to access the cubic nonlinearity of the SQUID. For the experiments presented here, we choose the ratio of the SQUID junction areas to be $1: 1.7$.

We would now like to understand how the external pump affects the cavity modes. We start by expanding $E_{J}\left(\hat{\Phi}_{\text {ext }}\right)$ to leading order in $\hat{\Phi}_{p}$ around the working point $\Phi_{\text {bias }}$. Assuming the pump to be a large-amplitude coherent state, we further apply the parametric approximation to the pump, representing it by the classical amplitude $\beta_{p}$. By now expanding the sinusoidal potential Eq. (1) in $\hat{\Phi}_{\text {cav }}$, we arrive at the approximate SQUID Hamiltonian

$$
\hat{H}_{\mathrm{SQ}} \approx \sum_{k} \hat{H}_{k}=\beta_{p} \sum_{k} \hbar g_{k}\left(\hat{a}_{1}+\hat{a}_{1}^{\dagger}+\hat{a}_{2}+\hat{a}_{2}^{\dagger}+\hat{a}_{3}+\hat{a}_{3}^{\dagger}\right)^{k},
$$

where the usual bosonic operators $\hat{a}_{i}$ and $\hat{a}_{i}^{\dagger}$ correspond to the three cavity modes considered, with resonant frequencies $f_{i}$. The familiar quadratic interactions, which produce TPDC, arise from keeping just the $k=2$ term of this expression [50], which is the lowest nonvanishing order if the SQUID is symmetric. For clarity here, we have omitted Kerr terms which are stationary in the lab frame, considering them instead in the Appendix A.

This Hamiltonian obviously contains a wide variety of quadratic, cubic, and higher-order terms. However, the presence of the pump gives us great flexibility in selectively enhancing the strength of certain desired terms. In the interaction picture, the various terms of $\hat{H}_{\mathrm{SQ}}$ have distinct time dependencies, oscillating at frequencies such as $3 f_{1}$ for the term $\hat{a}_{1}^{3}$. With the appropriate choice of pump frequency $f_{p}$ and the application of the rotating-wave approximation, we quickly see that we can selectively activate a desired set of parametric processes between the cavity modes, including cubic interactions (Appendix A 3). 
TABLE I. Cubic SPDC Hamiltonians explored in this work. Each row represents a distinct process and individual experiment, where we pump the SQUID at the specified frequency, driving three-photon down-conversion to one, two, or three cavity modes. We choose which Hamiltonian to explore simply by setting the pump frequency.

\begin{tabular}{llccccc}
\hline \hline & & \multicolumn{5}{c}{ Frequency [GHz] } \\
\cline { 2 - 5 } SPDC & \multicolumn{1}{c}{ Combinations } & Pump & Mode 1 & Mode 2 & Mode 3 & Effective Hamiltonians \\
\hline Single-mode & $f_{p 1}=3 \times f_{1}$ & 12.6 & 4.2 & $\ldots$ & $\ldots$ & $\hat{H}_{1 \mathrm{M}}=\hbar g\left(\hat{a}_{1}^{3}+\hat{a}_{1}^{\dagger 3}\right)$ \\
Two-mode & $f_{p 2}=2 \times f_{1}+f_{2}$ & 14.5 & 4.2 & 6.1 & $\ldots$ & $\hat{H}_{2 \mathrm{M}}=\hbar g\left(\hat{a}_{1}^{2} \hat{a}_{2}+\hat{a}_{1}^{\dagger 2} \hat{a}_{2}^{\dagger}\right)$ \\
Three-mode & $f_{p 3}=f_{1}+f_{2}+f_{3}$ & 17.8 & 4.2 & 6.1 & 7.5 & $\hat{H}_{3 \mathrm{M}}=\hbar g\left(\hat{a}_{1} \hat{a}_{2} \hat{a}_{3}+\hat{a}_{1}^{\dagger} \hat{a}_{2}^{\dagger} \hat{a}_{3}^{\dagger}\right)$ \\
\hline \hline
\end{tabular}

Importantly, as is standard in the parametric approximation, the terms with time dependencies that match the pump frequency have their strength enhanced by the pump amplitude; that is, they have an effective interaction strength $|g|=\left|\beta_{p}\right| g_{k}$. Together with cavity modes that can be easily designed, this device gives us a rich toolbox of both conventional and novel parametric processes, which can be explored with a high degree of flexibility. In this work, we limit our discussion to cubic Hamiltonians that produce three-photon SPDC, generating photons in one, two, and three modes. These Hamiltonians and their corresponding pump frequencies are shown in Table I (see Appendix A for derivations). We note that we observe other Hamiltonians, such as three-photon generalizations of beam-splitter interactions [51], but we do not explore them here.

\section{MEASUREMENTS}

\section{A. Three-photon SPDC to a single mode}

By pumping at the appropriate frequency, we experimentally realize the three cubic SPDC Hamiltonians listed in Table I. We measure the propagating output state of the cavity by first amplifying it with a cold HEMT amplifier and then a room-temperature amplifier chain. The system is absolutely calibrated using a shot noise tunnel junction, as described in detail in Ref. [41], giving calibrated values for the system gain, the system noise temperature, and the physical temperature of the input state. The amplified signal is split at room temperature, and the three modes are measured simultaneously using heterodyne detection, giving the field quadratures for the modes. In the rest of the paper, we denote the raw, room-temperature quadratures as $\hat{I}=\sqrt{G}\left(\hat{a}+\hat{a}^{\dagger}\right)$ and $\hat{Q}=-\sqrt{G} i\left(\hat{a}-\hat{a}^{\dagger}\right)$ and the calibrated quadratures, referred to the output of the cavity, as $\hat{x}=\hat{a}+\hat{a}^{\dagger}$ and $\hat{p}=-i\left(\hat{a}-\hat{a}^{\dagger}\right)$. The scaling factor $G$ includes the calibrated system gain. To quantify the down-converted signal emitted by the cavity, we define the photon flux density $F(\omega)$ defined by $\left\langle\hat{a}^{\dagger}(\omega) \hat{a}\left(\omega^{\prime}\right)\right\rangle=$ $F(\omega) \delta\left(\omega-\omega^{\prime}\right)$, which is the average number of photons per second per hertz propagating in the transmission line.

We start by exploring three-photon SPDC to a single cavity mode. We use the mode at approximately $f_{1}=4.2 \mathrm{GHz}$, by applying a pump tone at 3 times the cavity mode frequency, i.e., $f_{p}=12.6 \mathrm{GHz}$. This process activates the Hamiltonian $\hat{H}_{1 \mathrm{M}}$ in Table I. The effects of this single-mode Hamiltonian have been theoretically studied the most among the three Hamiltonians in Table I. Reference [19] predicts that phase-space interference between the down-converted photon triplets leads to a highly non-Gaussian Wigner function with the profile of a three-pointed star, with the three arms having triangular symmetry. Because of this distinct pattern, this single-mode trisqueezed state is also called a star state [16].

To look for experimental evidence of the star state, we start by simply examining phase-space histograms of the calibrated quadratures of the cavity output at $f_{1}$. The cavity input state is near vacuum, at a calibrated temperature of $30 \mathrm{mK}$. Figure 2 shows the results for two different pump strengths. Figure 2(a) shows the histogram of a strongly pumped state with $F=66$. For this bright state, the triangular symmetry of the generated state is readily apparent, even on top of the Gaussian system noise measured at $F=35$, illustrating the strong cubic interaction achieved. In Fig. 2(b), we also show data for a weakly pumped state with $F \approx 1$. In this case, the raw histogram is dominated by the system noise. However, the triangular symmetry of the state can still be observed when we subtract the (unpumped) system noise histogram from the pumped signal histogram.

Clearly, the phase-space distributions of our single-mode trisqueezed states are radically different from a two-photon Gaussian squeezed state, which we expect to lead to very different statistics. We use these statistics to make a more quantitative comparison between the theoretical predictions and our experimental results. Generally, we expect higherorder moments beyond the second-order variance and covariance to be significant. In particular, as we expect our interaction Hamiltonians to be cubic, three-point correlators are of interest. Accordingly, we quantify the nonGaussian character of our measured quadratures using the third standardized moment, also called the skewness, which for the random variable $y$ is given by $\gamma(y)=\left[\overline{(y-\bar{y})^{3}} / \sigma_{y}^{3}\right]$, where the overbar represents the expectation value, $\bar{y}$ is the mean, and $\sigma_{y}^{2}$ is the variance of $y$. (Below, we generally assume $\bar{y}=0$.) Roughly, the skewness measures the asymmetry of the distribution of $y$ and is zero for Gaussian variables. We note that, in what follows, we use the notational convention that, e.g., $x$ represents a classical 

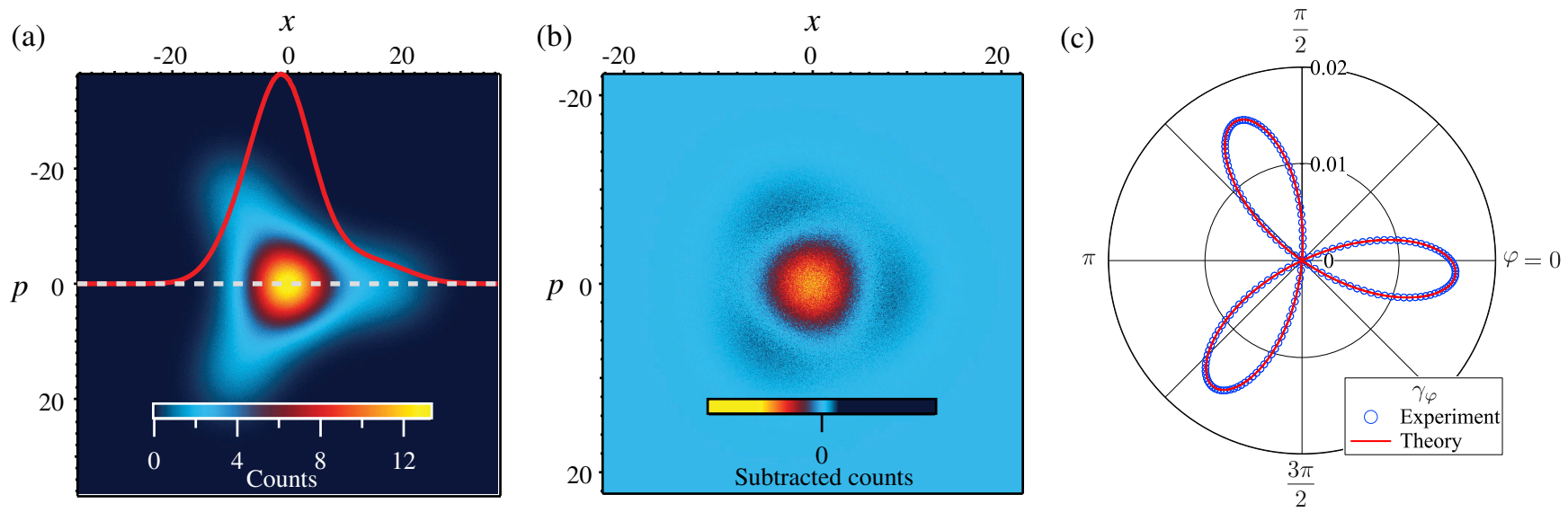

FIG. 2. Measured histograms of trisqueezed states in a single mode. (a) A histogram of a bright trisqueezed state with $F \approx 66$. The triangular symmetry of the histogram is apparent, clearly indicating its non-Gaussian character. The inset shows the projection of the $2 \mathrm{D}$ histogram onto the $x$ quadrature, which displays a significant amount of asymmetry (skewness), despite the system noise. (b) A histogram of a trisqueezed state with $F \approx 1$ after subtracting the system noise histogram. The triangular symmetry is still apparent despite the low photon flux. (c) A polar plot of the skewness $\gamma_{\varphi}$ of the generalized quadrature $x_{\varphi}$ (see the text) as a function of the phase angle $\varphi$, showing data and theory. The small rotation of $\gamma_{\varphi}$ is caused by a relative phase between the microwave pump and the local oscillator of the heterodyne receiver. The theory curve is normalized to the maximum experimental value and rotated appropriately but otherwise has no adjustable parameters. The plot is produced from the same raw data as (b) but without subtracting the system noise. This statistic essentially measures the asymmetry of the quadrature distribution with respect to the symmetry axis perpendicular to $x_{\varphi}$. We see that the triangular symmetry of the underlying state is very visible even though it is completely obscured in a histogram of the raw data, which suggests that $\gamma_{\varphi}$ is a good way to analyze the non-Gaussian character of our trisqueezed states. This approach is particularly effective because the skewness of the Gaussian system noise is zero (within our measurement error).

measurement record associated with the observable operator $\hat{x}$. Furthermore, we take quantities like $\left\langle x^{3}\right\rangle$ to represent classical time averages of measurement records, while $\left\langle\hat{x}^{3}\right\rangle$ represents the quantum correlator.

Looking at Fig. 2, it is clear that our measured quadrature distributions are asymmetric but also that the asymmetry is not invariant under phase rotations. That is, the skewness of $\hat{x}$ and $\hat{p}$ is not generally the same. To study the transformation properties of the skewness, we can define the generalized quadrature $\hat{x}_{\varphi}=\hat{x} \cos \varphi-\hat{p} \sin \varphi$. We can then study the skewness of the measurements of $\hat{x}_{\varphi}$, defining for simplicity $\gamma_{\varphi}=\gamma\left(x_{\varphi}\right)$. Essentially, $\gamma_{\varphi}$ measures the asymmetry of the quadrature distribution with respect to the symmetry axis perpendicular to the direction of $\hat{x}_{\varphi}$. We can also associate $\gamma_{\varphi}$ with the three-point quantum correlator $\left\langle\hat{x}_{\varphi}^{3}\right\rangle$. Figure 2(c) shows a polar plot of $\gamma_{\varphi}$ as a function of $\varphi$, for the data measured with $F \approx 1$. Unlike Fig. 2(b), we do not subtract the amplifier noise before calculating $\gamma_{\varphi}$, as the amplifier noise is expected to be symmetric and not contribute. We can make a number of comments on Fig. 2(c). First, we see that the signal-to-noise ratio in measuring $\gamma_{\varphi}$ is quite good, despite it being a higher-order statistic. Second, we see that the triangular symmetry of the underlying state is quite apparent even though, in the raw data, it is completely obscured by the amplifier noise. Next, we can conclude from the strong nodes near $\varphi=(2 \pi / 3)(n+1 / 2)$ that the skewness of the amplifier noise is indeed small. Finally, we can observe that the nodes correspond to angles where the symmetry plane aligns with a lobe of the star, while the antinodes correspond to the symmetry plane being perpendicular to a lobe. Overall, we observe that measuring $\gamma_{\varphi}$ appears to be a useful way to characterize the non-Gaussian character of the single-mode trisqueezed states.

\section{B. Three-photon SPDC to multiple modes}

Now, we look at multimode trisqueezed states. As previously mentioned, this includes two-mode states and three-mode states. Once again, these states can be produced by the appropriate choice of the pump frequency, naturally given by the conservation of energy (see Table I). Similar to the single-mode trisqueezed state, we expect significant third-order statistics in the output states. Thus, we statistically characterize the two-mode and three-mode trisqueezed states using the so-called coskewness of $A, B, C$ defined as $\gamma_{A B C}=\overline{A B C} /\left(\sigma_{A} \sigma_{B} \sigma_{C}\right)$, here assuming the measurements are mean zero. Now, we can associate this statistical measure with the three-point quantum correlators $\langle\hat{A} \hat{B} \hat{C}\rangle$, where $\hat{A}, \hat{B}$, and $\hat{C}$ are some quadratures of the multimode trisqueezed states.

While pumping, the amplified output signal of the cavity is split at room temperature and the three modes measured simultaneously by three digitizers to obtain the multimode field quadrature data. Motivated by standard two-photon SPDC, we start by looking for covariance between each pair of modes. However, we observe no covariance 
between any pair of the modes in either the two-mode or three-mode case, as shown in Fig. 3. Instead, we find significant coskewness between the signals (see Figs. 3 and 4). To verify that the observed coskewness is a coherent process related to the pump, we sweep the pump phase and compute the covariances and coskewnesses, as shown in Fig. 3 for both the two-mode and three-mode cases. We see that the coskewness oscillates with the pump phase between positive and negative extremes, while the covariance remains unchanged at zero. This observation of coskewness (three-body interactions) in the absence of covariance (two-body interactions) is a striking and extremal observation of non-Gaussian statistics in both the two-mode and three-mode cases. Furthermore, in the three-mode case, it clearly demonstrates three-photon interference, which has only very recently been observed in any system $[37,38]$.

With a total of six quadratures, there are a number of two-mode and three-mode coskewnesses that we can
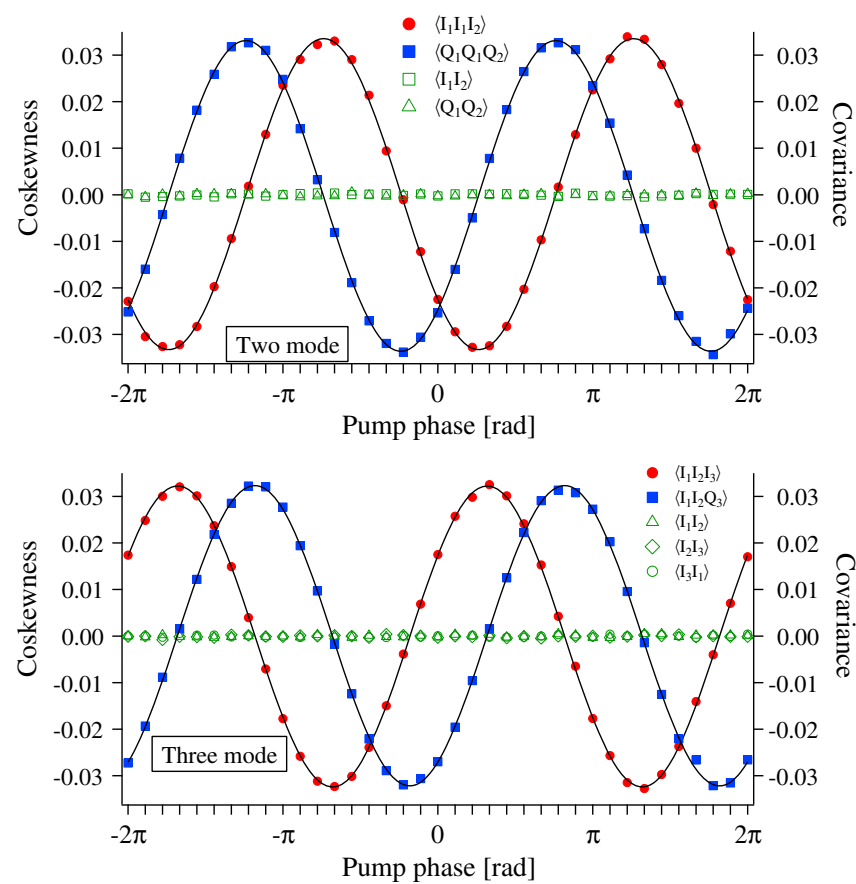

FIG. 3. Covariance and coskewness of three-photon SPDC to (top) two modes and (bottom) three modes measured at room temperature. In the two-mode case, we have a degeneracy between two of the three generated photons. This degeneracy leads to one mode participating twice in the nonzero coskewness term. For our choice of pump phase, the only significant coskewness terms are then $\left\langle I_{1}^{2} I_{2}\right\rangle$ and $\left\langle Q_{1}^{2} I_{2}\right\rangle$. In the three-mode case, the only nonzero coskewness term must contain all three modes, e.g., $\left\langle I_{1} I_{2} I_{3}\right\rangle$. To show that the observed coskewness is coherently generated by the pump, we sweep the pump phase from $-2 \pi$ to $2 \pi$ and observe the effect. We see a clear oscillation in the coskewness. The oscillations are fit well by a sinusoid. Meanwhile, all covariance terms are essentially zero throughout the sweep, indicating that the generated states do not contain second-order correlations. compute. Figure 4 shows several of them. In the two-mode case, we have a degeneracy between two of the three generated photons; that is, two photons will go to one of the modes. Therefore, we expect that only terms such as $\left\langle I_{1}^{2} I_{2}\right\rangle$, where one mode participates twice in the coskewness, will be nonzero. In the three-mode case, where one photon goes to each mode, we expect that the only nonzero coskewness terms should contain all three modes, e.g., $\left\langle I_{1} I_{2} I_{3}\right\rangle$. This prediction is exactly what is observed.

In an attempt to impose structure on the myriad threebody correlators, we now look to generalize the phase rotation analysis used in Fig. 2(c). We take as transformations to study the set of symmetry operations of an $\mathrm{N}$-mode Gaussian state, which forms the symplectic group [52], and are generated by quadratic Hamiltonians. The symplectic group includes squeezing operations which create or destroy photons, but we restrict ourselves to the passive operations of the group. These operations form a unitary subgroup and include phase rotations of a single mode, with generators such as $\hat{a}_{i}^{\dagger} \hat{a}_{i}+\hat{a}_{i} \hat{a}_{i}^{\dagger}$, and beam-splitter rotations between modes, with generators such as $\hat{a}_{i}^{\dagger} \hat{a}_{j}+\hat{a}_{i} \hat{a}_{j}^{\dagger}$. Importantly, we expect different cubic Hamiltonians to have different transformation properties under these operations.
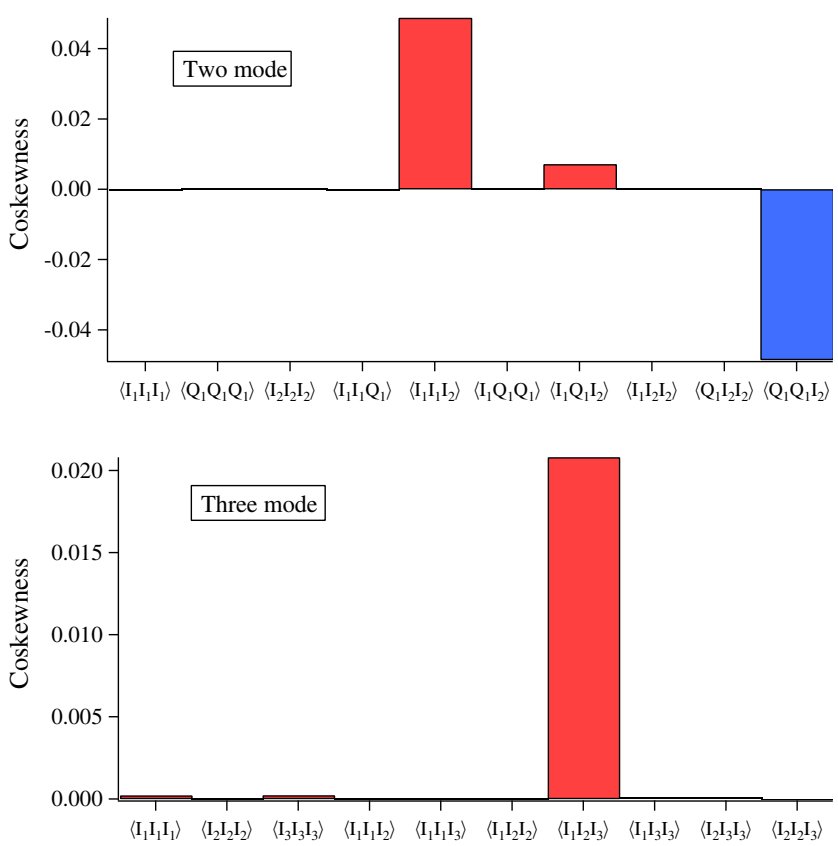

FIG. 4. Measured coskewness in (top) the two-mode case and (bottom) the three-mode case. In the two-mode case, we consider the three quadratures $\hat{I}_{1}, \hat{Q}_{1}$, and $\hat{I}_{2}$ as a representative sample. The pump frequency is chosen such that mode 1 is the degenerate mode. Accordingly, we expect only $\left\langle I_{1}^{2} I_{2}\right\rangle,\left\langle Q_{1}^{2} I_{2}\right\rangle$, and $\left\langle I_{1} Q_{1} I_{2}\right\rangle$ to be nonzero, which is what is observed. Their relative values are determined by the pump phase [Eq. (B5)]. In the three-mode case, we consider the $\hat{I}$ quadratures of all three modes. As expected, the only nonzero term includes all three modes, i.e., $\left\langle I_{1} I_{2} I_{3}\right\rangle$. 
With three modes, we have six quadratures, implying a 6D phase space to explore. The symplectic operations can then be represented by $6 \times 6$ matrices, which can be written as transformations between the quadratures. In order to illustrate the transformation properties with a 3D figure, we project into a three-quadrature subspace. The transformations then become generalized rotations between the three chosen quadratures. Collecting the quadratures into a 3-vector, we can explore arbitrary combinations of the quadratures by apply a series of two rotations according to

$$
\left(\hat{A}_{\phi \theta}, \hat{B}_{\phi \theta}, \hat{C}_{\phi \theta}\right)^{t}=R_{C}(\phi) \times R_{B}(\theta) \times(\hat{A}, \hat{B}, \hat{C})^{t},
$$

where $R_{i}$ are standard 3D rotation matrices with the rotation axis specified by the subscript. After the rotations, we consider the generalized quadrature

$$
\hat{A}_{\phi \theta}=\cos (\phi) \cos (\theta) \hat{A}-\sin (\phi) \hat{B}+\cos (\phi) \sin (\theta) \hat{C}
$$

and compute its skewness $\gamma_{\phi \theta}$, which, in general, is a mix of all of the possible three-point correlators of the three quadratures. Generalizing the one-mode case, we can think of $\gamma_{\phi \theta}$ representing the asymmetry of the 3D distribution of measured quadratures with respect to the symmetry plane perpendicular to the direction of $\hat{A}_{\phi \theta}$.

In Fig. 5, we show spherical plots of $\gamma_{\phi \theta}$ as a function of $\theta \in[0, \pi]$ and $\phi \in[0,2 \pi]$ for both the two-mode and

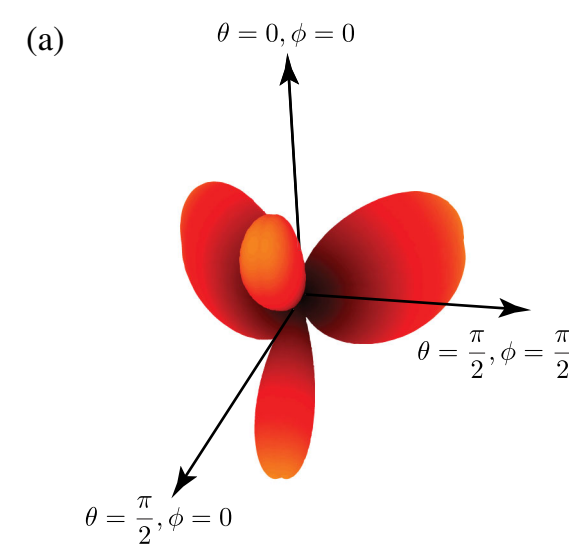

(d)

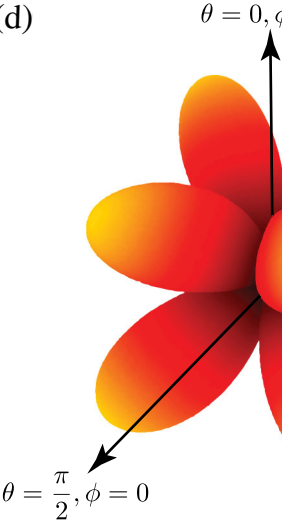

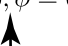

$\phi=0$
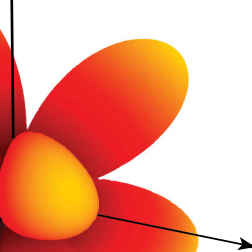

(b)

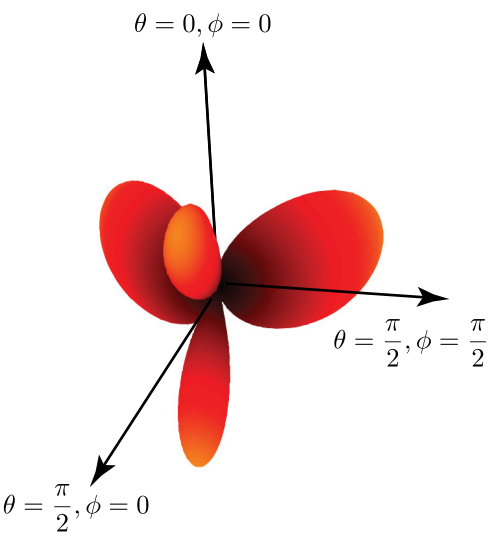

(e)

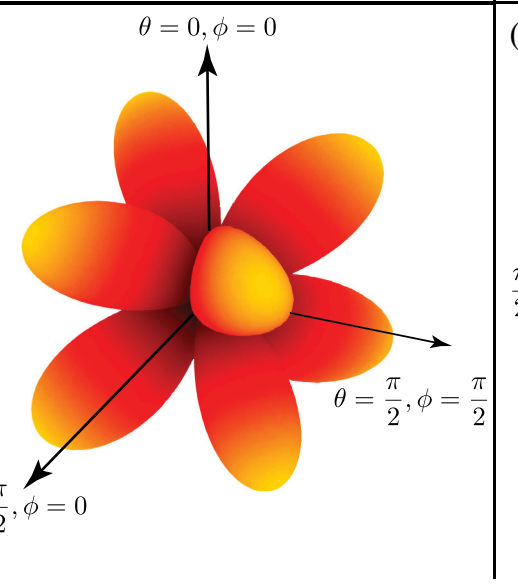

(c)

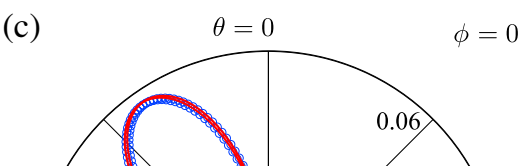

$\frac{\pi}{2}$
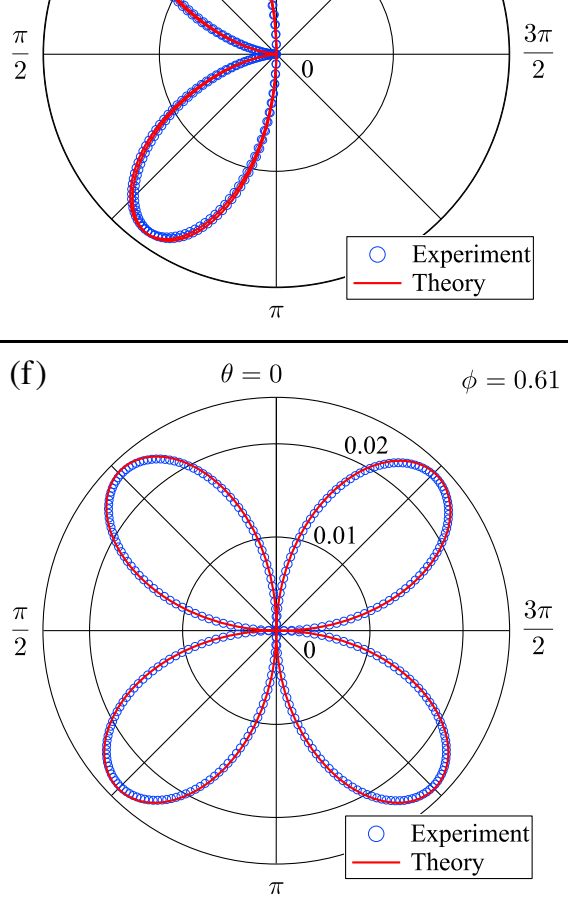

FIG. 5. Third-order correlation analysis of multimode trisqueezed states. Rows 1 and 2 show results for, respectively, the two-mode and three-mode trisqueezed states, generated by $\hat{H}_{2 \mathrm{M}}$ and $\hat{H}_{3 \mathrm{M}}$. The spherical plots show $\gamma_{\phi \theta}$, which is the skewness of the generalized multimode quadrature $\hat{A}_{\phi \theta}$ [see Eq. (6)], which mixes the mode quadratures through symplectic symmetry operations. The 6D phase space of the three modes is projected into a 3D space for the purposes of visualization. Generally, $\gamma_{\phi \theta}$ combines the contributions of the skewness and coskewness of the three modes involved in each case. (a) Experimental data for the two-mode case, with a maximum skewness of 0.057. (b) The theoretical prediction for the two-mode cubic Hamiltonian $\hat{H}_{2 \mathrm{M}}$. The clear agreement shows that the observed state is generated by that specific Hamiltonian. (c) A plane cut of the spherical plots through the plane at $\phi=0$. The theory curve is normalized to the maximum experimental value, but otherwise the theory has no adjustable parameters. There are no lobes apparent on the right half of the plot, because $\gamma_{\phi \theta}$ is negative for $\phi \in[\pi / 2,3 \pi / 2]$, causing these lobes to overlap those of $\phi \in[-\pi / 2, \pi / 2]$. (d) Experimental data for the three-mode case, with a maximum skewness of 0.024. (e) The theoretical prediction for the three-mode cubic Hamiltonian $\hat{H}_{3 \mathrm{M}}$. (f) A plane cut of the spherical plots through the plane at $\phi=0.61\left(35^{\circ}\right)$. Again, we see a clear agreement between the observed state and the target Hamiltonian. In particular, the antinodes (lobes) of $\gamma_{\phi \theta}$ appear only at angles where all three modes are mixed, as expected for genuine three-mode interference. 
three-mode cases. Explicitly, for the two-mode case, we chose $A=I_{1}, B=Q_{1}$, and $C=I_{2}$. The transformation specified by Eq. (5) is then first a beam-splitter rotation by $\theta$ between mode 1 and mode 2 , followed by a phase rotation of mode 1 by $\phi$ (which mixes $I$ and $Q$ ). In the three-mode case, we have $A=I_{1}, B=I_{2}$, and $C=I_{3}$, such that Eq. (5) describes two beam-splitter rotations among the three modes, first coupling mode 1 and mode 3 and then modes 1 and 2. Figure 5 shows the experimental results for $\gamma_{\phi \theta}$ along with theoretical predictions for the states produced by the Hamiltonians $\hat{H}_{2 \mathrm{M}}$ and $\hat{H}_{3 \mathrm{M}}$ in Table I. We include details of the theoretical predictions in Appendix B. We remark that the agreement between the theory and experiment is very good. We see that different processes produce very different shapes for $\gamma_{\phi \theta}$, which usefully fingerprint the underlying Hamiltonians and allow us to see in a clear, visual way how cleanly we generate one Hamiltonian compared to another.

As an example, we can consider the three-mode case. A first important feature we note is that $\gamma_{\phi \theta}$ has nodes in the three planes defined by the pairs of axes, that is, the planes where we alternately fix $\phi=0, \phi=\pi / 2$, and $\theta=\pi / 2$. We can understand the presence of these nodes by noticing that in these planes at least one of the modes is excluded from the generalized quadrature $\hat{A}_{\phi \theta}$. That is, this observation tells us that two-mode correlators such as $\left\langle I_{1}^{2} I_{2}\right\rangle$ and singlemode correlators such as $\left\langle I_{1}^{3}\right\rangle$ are zero, exactly as we would expect for a state generated by the pure three-mode Hamiltonian $\hat{H}_{3 \mathrm{M}}$. Conversely, the antinodes (lobes) correspond to the angles where the contribution of the threemode correlator $\left\langle I_{1} I_{2} I_{3}\right\rangle$ are maximized. The pattern therefore tells us both that we have activated $\hat{H}_{3 \mathrm{M}}$ and that we have not activated $\hat{H}_{1 \mathrm{M}}$ nor $\hat{H}_{2 \mathrm{M}}$. We note separately that this is strong evidence that we are observing genuine three-mode interference.

We can understand the structure of the two-mode state in Fig. 5(a) in a similar way. First, recall that, for our specific choice of pump frequency (see Table I), we expect that we are creating two photons in mode 1 and one photon in mode 2 . We can then consider the behavior in the $\phi=0$ plane, also highlighted in Fig. 5(c). We see clear nodes at $\theta=0(\pi / 2)$, where we are calculating the single-mode skewness of $\hat{I}_{1}\left(\hat{I}_{2}\right)$ alone, in agreement with our expectations. Instead, $\gamma_{\phi \theta}$ is maximum around $\theta=\pi / 4$ and $\theta=3 \pi / 4$, where $\hat{I}_{1}$ and $\hat{I}_{2}$ are maximally mixed, and we get the maximum contribution from the two-mode correlator $\left\langle I_{1}^{2} I_{2}\right\rangle$. As above, this fingerprint nicely indicates that we are, in this case, activating $\hat{H}_{2 \mathrm{M}}$ but not $\hat{H}_{1 \mathrm{M}}$. We note that the expected lobes at $\theta=5 \pi / 4$ and $\theta=7 \pi / 4$ are missing at first glance, but, in fact, they overlap the lobes at $\theta=\pi / 4$ and $\theta=3 \pi / 4$, because the sign of $\gamma_{\phi \theta}$ becomes negative. The structure in the $\theta=\pi / 2$ plane can be explained in a similar way, except there we are mixing $\hat{Q}_{1}$ and $\hat{I}_{2}$.
We can generate several more of these projections of the $6 \mathrm{D}$ phase space into $3 \mathrm{D}$, but it is already clear from these two examples that the structure of $\gamma_{\phi \theta}$ is a useful way to characterize the output state. In particular, with a library of the possible forms generated by different cubic Hamiltonians, we can quickly see which one is generating the observed state. By observing the relative depth of nodes compared to the antinodes, we can also appreciate how purely we generate just a single member of the cubic Hamiltonian family. For instance, for the three-mode data shown in Fig. 5(d), this ratio is approximately $10^{-4}$, indicating a high degree of purity.

\section{Correlation feed-forward}

In this section, we explore more deeply the striking feature that our observed three-photon states exhibit threemode correlations (skewness) in the absence of two-mode correlations (covariance), which is in strong contrast to the conventional two-photon states and Gaussian states generally. An interesting experimental observation in this direction is that, while the three-mode trisqueezed state does not show covariance between any pairs of modes when starting from a vacuum state, we observe that by seeding one of the three modes with a weak coherent tone the noise power emitted from the other modes is enhanced and that it then has nonzero covariance. Similarly, in the two-mode trisqueezed state, by seeding the mode participating only once, i.e., mode 2 , we observe the emission of squeezed noise from the other mode. These results can be understood from the point of view of dynamical constraints imposed by the conservation of energy. In the standard twophoton case, signal and idler photon pairs are constrained to have a symmetric detuning around $f_{p} / 2$. In the rotating frame at $f_{p} / 2$, the phasors of the signal and idler photons precess at the same frequency, but in opposite directions, such that the axis (phase) of the sum phasor is stationary in time and the same for all pairs. This preferred phase gives rise to the observed covariance between the signal and idler modes. In the three-photon case, we have three free frequencies (energies) with no fixed relation between them for any pair of photons, washing out the two-mode correlations. Seeding effectively fixes one photon frequency in the resulting stimulated emission, leaving a fixed relation between the other two, which produces correlations similar to two-photon SPDC.

This argument suggests that, by having information about one mode, it should be possible to reveal the twomode correlation of the remaining modes. That is, by conditioning our measurements of the remaining modes on the measurement of the first "reference" mode, we should be able recover a conditional distribution with two-mode correlations. To validate our hypothesis, we perform the following "correlation feed-forward" protocol. First, we estimate the phase of the reference mode for every sample period using the standard relation $\phi_{\text {ref }}=\tan ^{-1}\left(Q_{\text {ref }} / I_{\text {ref }}\right)$. 


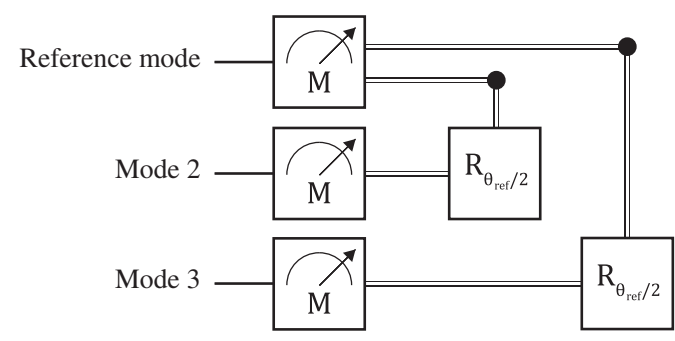

FIG. 6. A cartoon of the correlation feed-forward protocol for the three-mode trisqueezed state. This scheme tests our hypothesis on the conditional structure of two- and three-mode correlations. In the three-mode protocol shown here, we first measure the local phase of the reference mode (mode 1 in this example), followed by applying a rotation by $\phi_{\text {ref }} / 2$ on the other two modes. The result is an observable correlation between mode 2 and mode 3 with the characteristic structure of two-photon SPDC, i.e., twomode squeezing.

(Our data are sampled at $1 \mathrm{MHz}$, corresponding to an integration time of $1 \mu \mathrm{s}$.) We then rotate the quadratures of the remaining modes using $\phi_{\text {ref }}$, as illustrated in Fig. 6. The action of the phase rotation can again be explained by energy conservation: as the frequencies of the three photons must sum to $f_{p}$, a small frequency shift $\left(\delta f_{\text {ref }}\right)$ from the center in the reference mode needs to be compensated by changes in the other two modes. In the three-mode case, we use mode 1 as the reference and apply rotations by $\phi_{1} / 2$ to modes 2 and 3. Table II shows the resulting correlations recovered.

We also apply the protocol to the two-mode trisqueezed state, using mode 2 as the reference. After doing this process, we see squeezing effects in mode 1 with a ratio in the variance of $\hat{x}$ to $\hat{p}$ of 1.11 , compared to 1.00 without the feed-forward. To visualize the effect of the correlation feed-forward protocol, in Fig. 7 we show a histogram of the corrected quadratures after subtracting the system noise histogram. We can clearly see a stretching of the distribution along the $\hat{x}$ quadrature, indicating squeezinglike correlations.

TABLE II. The resultant two-mode correlation coefficient (normalized covariance) between mode 2 and mode 3 after applying correlation feed-forward to the three-mode trisqueezed state (see Fig. 6). As shown, we recover a significant amount of correlation when applying our feed-forward correction. We include the results of applying the protocol to the system noise with the pump off to verify that the protocol itself does not introduce spurious correlations.

\begin{tabular}{|c|c|c|c|c|}
\hline \multirow[b]{3}{*}{ Quads } & \multicolumn{4}{|c|}{ Feed-forward } \\
\hline & \multicolumn{2}{|c|}{ Yes } & \multicolumn{2}{|c|}{ No } \\
\hline & Pump on & Pump off & Pump on & Pump off \\
\hline$I_{2} I_{3}$ & $0.30 \pm 0.02$ & $0.00 \pm 0.02$ & $0.00 \pm 0.02$ & $0.00 \pm 0.02$ \\
\hline$Q_{2} Q_{3}$ & $-0.30 \pm 0.02$ & $0.00 \pm 0.02$ & $0.00 \pm 0.02$ & $0.00 \pm 0.02$ \\
\hline$I_{2} Q_{3}$ & $-0.36 \pm 0.02$ & $0.00 \pm 0.02$ & $0.00 \pm 0.02$ & $0.00 \pm 0.02$ \\
\hline$Q_{2} I_{3}$ & $-0.36 \pm 0.02$ & $0.00 \pm 0.02$ & $0.00 \pm 0.02$ & $0.00 \pm 0.02$ \\
\hline
\end{tabular}

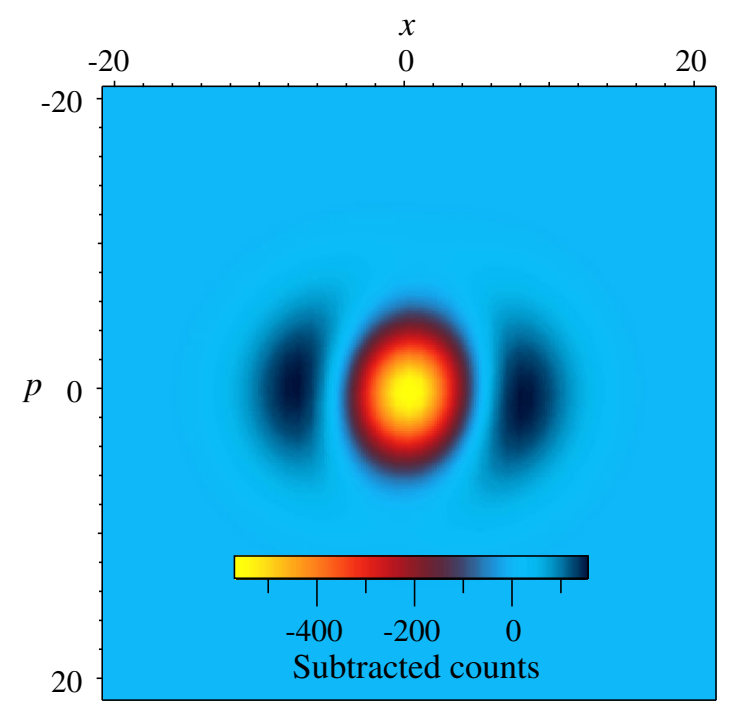

FIG. 7. The resultant single-mode histogram of mode 1 after applying the correlation feed-forward protocol to the two-mode trisqueezed state. We take mode 2 as the reference mode, correcting the phase of mode 1 . Then, we compute the histogram of the data and subtract from it the histogram of the system noise (with the pump turned off) after also applying the protocol. We clearly see that the subtracted histogram is stretched along the $x$ axis, indicating squeezing of the on state. The small rotation of the figure can be explained by a small misalignment of the pump and digitizer phases (see Fig. 4).

These results validate our hypothesis about the conditional structure of two-mode and three-mode correlations in our system. At the same time, the correlation feed-forward demonstrations here are only proofs of principle, because our phase measurements are strongly contaminated by the system noise. In future work, it would be interesting to explore the fundamental limits of this reconstruction method.

\section{CONCLUSION}

In this work, we demonstrate a device which implements efficient three-photon SPDC in the microwave domain. The device is highly flexible, allowing us to perform singlemode, two-mode, and three-mode SPDC by simply selecting the appropriate pump frequency. We carefully compare the observations of various features of the three-photon SPDC states, verifying that our output signal is generated by the chosen interaction Hamiltonian. By extending parametric interactions to higher order, our device opens up novel possibilities that will enable a new wave of novel experimental and theoretical studies in microwave quantum optics.

\section{ACKNOWLEDGMENTS}

The authors thank B. Plourde, J. J. Nelson, and M. Hutchings at Syracuse University for invaluable help in 
junction fabrication. We also thank J. Aumentado of NISTBoulder for providing the SNTJ calibration source. Finally, we thank B. Sanders for useful discussions and insight. C. M. W., C. W. S. C., I. N., and A. M. V. acknowledge the Canada First Research Excellence Fund (CFREF), NSERC of Canada, the Canadian Foundation for Innovation, the Ontario Ministry of Research and Innovation, and Industry Canada for financial support. C. S. has received financial support through the Postdoctoral Junior Leader Fellowship Program from la Caixa Banking Foundation (LCF/BQ/ LR18/11640005). P.F.-D. acknowledges support of a fellowship from "la Caixa Foundation" (ID100010434) with code LCF/BQ/PR19/11700009 and funding from the Ministry of Economy and Competitiveness, through Contracts No. FIS2017-89860-P and Severo Ochoa No. SEV-2016-0588. F. Q. and G. J. acknowledge the Knut and Alice Wallenberg Foundation.

\section{APPENDIX A: ENGINEERING CUBIC INTERACTIONS IN CIRCUIT QED}

In these notes, we explore the possibility of engineering three-photon processes in a superconducting circuit architecture. For this purpose, we exploit the asymmetry between the two junctions of a dc SQUID as well as the resonant selection of terms of a Hamiltonian by means of a time-dependent bias on the SQUID. For simplicity, we drop the hats from the quantum operators in the Appendixes.

\section{Asymmetric SQUID}

Consider the circuit depicted in Fig. 8. The Lagrangian describing it is

$$
L_{\mathrm{SQUID}}=\sum_{j=1}^{2}\left[\frac{C_{J, j}}{2} \dot{\Phi}_{j}^{2}+E_{J, j} \cos \left(2 \pi \frac{\Phi_{j}}{\Phi_{0}}\right)\right],
$$

with $E_{J, 1} \neq E_{J, 2}, C_{J, 1} \neq C_{J, 2}$, and $\Phi_{0}=h / 2 e$ the flux quantum. One of the flux variables can be eliminated by means of the fluxoid quantization condition:

$$
\Phi_{\mathrm{ext}}=\Phi_{2}-\Phi_{1}
$$

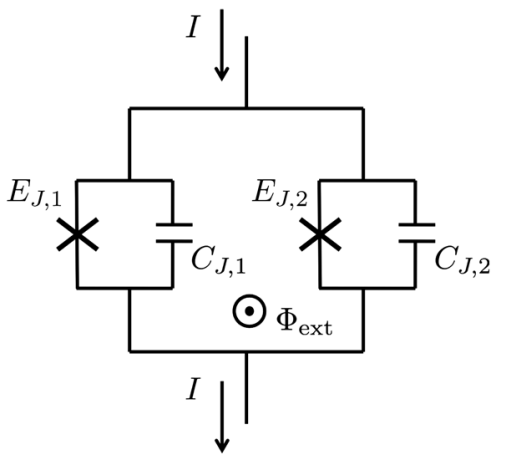

FIG. 8. Lumped-element description of a dc SQUID.
We now introduce the change of variables

$$
\begin{aligned}
& \Phi_{1}=\Phi-\frac{\Phi_{\mathrm{ext}}}{2}, \\
& \Phi_{2}=\Phi+\frac{\Phi_{\mathrm{ext}}}{2} .
\end{aligned}
$$

Rewriting Eq. (A1) in terms of Eqs. (A2) and (A3), we arrive to

$$
L_{\mathrm{SQUID}}=\frac{C_{T}}{2} \dot{\Phi}^{2}+E_{J}\left(\Phi_{\mathrm{ext}}\right) \cos \left(2 \pi \frac{\Phi}{\Phi_{0}}-\alpha\right)
$$

with $C_{T}=C_{J, 1}+C_{J, 2}$,

$$
E_{J}\left(\Phi_{\mathrm{ext}}\right)=\sqrt{E_{J, 1}^{2}+E_{J, 2}^{2}+2 E_{J, 1} E_{J, 2} \cos \left(2 \pi \frac{\Phi_{\mathrm{ext}}}{\Phi_{0}}\right)},
$$

and

$$
\alpha=\arctan \left[\tan \left(\pi \frac{\Phi_{\mathrm{ext}}}{\Phi_{0}}\right) \frac{E_{J, 1}-E_{J, 2}}{E_{J, 1}+E_{J, 2}}\right] .
$$

We can find a simplified, approximate form for $E_{J}$ by first defining $E_{J, 1}=E$ and $E_{J, 2}=E+\delta$ along with $\Delta=\delta / 2 E$. To first order in $\Delta$, we find

$$
\begin{aligned}
& \frac{E_{J, 1}-E_{J, 2}}{E_{J, 1}+E_{J, 2}}=\Delta+O\left(\Delta^{2}\right), \\
& E_{J}\left(\Phi_{\mathrm{ext}}\right)^{*} \simeq 2 E \sqrt{1+2 \Delta}\left|\cos \left(\frac{\Phi_{\mathrm{ext}}}{2 \varphi_{0}}\right)\right|,
\end{aligned}
$$

which has a form similar to a symmetric SQUID. For $\Phi_{\text {ext }} / \varphi_{0}$ around $\pi / 4$, moderate asymmetries of $\Delta \simeq 0.2$ give errors smaller than $3 \%$.

\section{Transmission line resonator +SQUID Lagrangian}

From Eq. (A4), the Lagrangian for a transmission line resonator of length $d$ terminated by a SQUID placed at $x=d$ (see Fig. 9) is

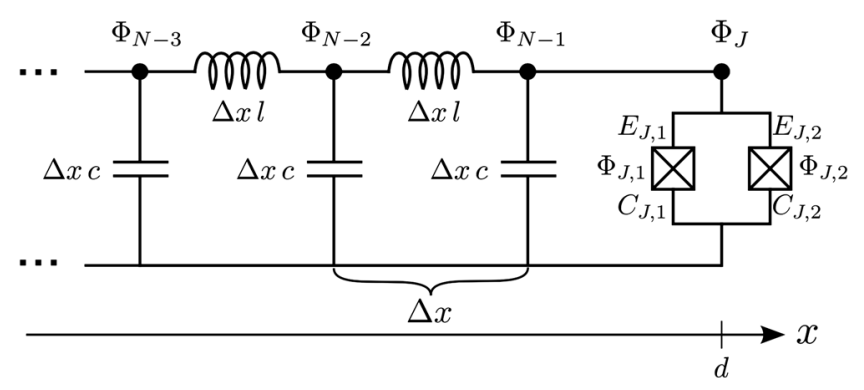

FIG. 9. Lumped-element description of a transmission line resonator terminated by a SQUID (adapted from Ref. [50]). 


$$
\begin{aligned}
L= & \frac{1}{2} \int_{0}^{d} d x\left\{c[\dot{\Phi}(x, t)]^{2}-l^{-1}\left[\partial_{x} \Phi(x, t)\right]^{2}\right\} \\
& +E_{J}\left[\Phi_{\text {ext }}(t)\right] \cos \left(\frac{\Phi(d, t)}{\varphi_{0}}-\alpha(t)\right)
\end{aligned}
$$

with $c$ and $l$ the capacitance and inductance per unit length of the resonator, respectively. In general, the external flux $\Phi_{\text {ext }}$ threading the SQUID does not need to be static. Following Eq. (A6), a time-dependent external signal renders the phase $\alpha$ time dependent as well.

Notice that if $\alpha=$ const, we can redefine the field as $\Phi(x, t) \rightarrow \Phi(x, t)+\varphi_{0} \alpha$ in order to get rid of the shift in the cosine potential. This transformation leaves the resonator Lagrangian invariant.

Now, consider the following time-dependent functions:

$$
\begin{aligned}
\Phi_{\mathrm{ext}}(t) & =\Phi_{\mathrm{ext}}^{0}+\delta \Phi_{\mathrm{ext}}(t), \\
\alpha(t) & =\alpha^{0}+\delta \alpha(t) .
\end{aligned}
$$

In both cases, $\left|\delta \Phi_{\text {ext }}(t)\right| / \Phi_{\text {ext }}^{0},|\delta \alpha(t)| / \alpha^{0} \ll 1$. Once again, we can redefine the field as $\Phi(x, t) \rightarrow \Phi(x, t)+\varphi_{0} \alpha^{0}$, i.e., shift it around the constant offset $\alpha^{0}$. Nevertheless, this time we are left with the small (time-dependent) fluctuations $\delta \alpha(t)$ around $\alpha^{0}: \cos \left[\Phi(d, t) / \varphi_{0}-\delta \alpha(t)\right]$.

Following our discussion in the previous section, we can approximate the Josephson energy by Eq. (A7). In addition, from Eq. (A8) we can write

$$
\frac{\Phi_{\mathrm{ext}}(t)}{2 \varphi_{0}}=\bar{\varphi}+\lambda g(t)
$$

with $\lambda$ assumed to be small.

Thus, up to first order in $\lambda$ we have

$$
\cos \left(\frac{\Phi_{\mathrm{ext}}(t)}{2 \varphi_{0}}\right) \simeq \cos (\bar{\varphi})-\lambda \sin (\bar{\varphi}) g(t),
$$

and

$$
E_{J}\left[\Phi_{\mathrm{ext}}(t)\right] \simeq \bar{E}[1-\lambda \tan \bar{\varphi} g(t)],
$$

where we define $\bar{E}=2 E \sqrt{1+2 \Delta} \cos \bar{\varphi}$.

Similarly, from Eqs. (A6) and (A10),

$\alpha(t) \simeq \arctan [\tan (\bar{\varphi}) \Delta]+\lambda \frac{\sec ^{2} \bar{\varphi} \Delta}{1+[\tan (\bar{\varphi}) \Delta]^{2}} g(t)$.

We identify the second term on the right-hand side of Eq. (A11) with $\delta \alpha(t)$ :

$$
\delta \alpha(t)=\lambda \frac{\sec ^{2} \bar{\varphi} \Delta}{1+[\tan (\bar{\varphi}) \Delta]^{2}} g(t) .
$$

From now on, we write the latter as $\delta \alpha(t)=\lambda \delta \bar{\alpha} g(t)$. For our experimental SQUID asymmetry of $\Delta=0.26$, a typical working point of $\bar{\varphi}=0.045 \pi$ gives $\delta \bar{\alpha}=0.26$.

So far, we have considered a perturbative expansion in small oscillations of the external flux threading the SQUID loop. Now, we expand in terms of small oscillations of the resonator flux at the SQUID position $\Phi(d, t) \equiv \Phi_{J}$ : $\Phi_{J} / \varphi_{0} \ll 1$. Expanding the cosine potential as

$$
\begin{aligned}
& \cos \left(\frac{\Phi_{J}}{\varphi_{0}}-\delta \alpha\right) \\
& =1-\frac{1}{2 !}\left(\frac{\Phi_{J}}{\varphi_{0}}-\delta \alpha\right)^{2}+\frac{1}{4 !}\left(\frac{\Phi_{J}}{\varphi_{0}}-\delta \alpha\right)^{4}+O\left(\frac{\Phi_{J}}{\varphi_{0}}-\delta \alpha\right)^{6} \\
& \simeq 1-\frac{1}{2 !}\left(\frac{\Phi_{J}}{\varphi_{0}}\right)^{2}+\delta \alpha\left(\frac{\Phi_{J}}{\varphi_{0}}\right)+\frac{1}{24}\left(\frac{\Phi_{J}}{\varphi_{0}}\right)^{4}+\frac{1}{6} \delta \alpha\left(\frac{\Phi_{J}}{\varphi_{0}}\right)^{3}
\end{aligned}
$$

where we neglect terms of the order of $O\left(\lambda^{2}\right)$ and $O\left[\left(\Phi_{J} / \varphi_{0}\right)^{5}\right]$. In this way, we get the total Lagrangian in the small fluctuations and amplitude approximation

$$
L=L_{0}+L_{1},
$$

with $L_{0}$ the linear part

$$
\begin{aligned}
L_{0}= & \frac{1}{2} \int_{0}^{d} d x\left\{c[\dot{\Phi}(x, t)]^{2}-l^{-1}\left[\partial_{x} \Phi(x, t)\right]^{2}\right\} \\
& +\lambda \bar{E}(\delta \bar{\alpha}) g(t)\left(\frac{\Phi_{J}}{\varphi_{0}}\right)-\frac{1}{2} \bar{E}[1-\lambda \tan \bar{\varphi} g(t)]\left(\frac{\Phi_{J}}{\varphi_{0}}\right)^{2}
\end{aligned}
$$

and $L_{1}$ the nonlinear or interacting part

$$
L_{1}=\frac{1}{6} \lambda \bar{E}(\delta \bar{\alpha}) g(t)\left(\frac{\Phi_{J}}{\varphi_{0}}\right)^{3}+\frac{1}{24} \bar{E}[1-\lambda \tan \bar{\varphi} g(t)]\left(\frac{\Phi_{J}}{\varphi_{0}}\right)^{4} .
$$

Following the standard procedure [53], we first diagonalize Eq. (A12). We then reintroduce the linear normal mode decomposition into $L_{1}$. This last step is valid only whenever the energy of the nonlinear terms is much smaller than the normal modes' frequencies.

\section{a. Linear Lagrangian}

Up to second order in the flux amplitude, the resonator terminated by a SQUID is described by the Lagrangian $L_{0}$. The SQUID contributes with both a linear and a quadratic term. The quadratic term can be understood as an inductive contribution. The linear term corresponds to a classical drive. This correspondence is an undesirable consequence of breaking the SQUID symmetry; however, this term can safely be neglected in the operation regimes explored here (because the pump frequency is off resonant with the resonator modes). 
As a first approximation, we consider the normal modes of the system in the absence of a modulation, i.e., $\lambda=0$. In this regime, the field in the resonator satisfies the wave equation, which, in turn, can be solved using a separation of variables ansatz:

$$
\Phi(x, t)=\sum_{n=1}^{\infty} \phi_{n}(t) \cos \left(k_{n} x\right) .
$$

The boundary condition (current conservation) at the position of the SQUID $(x=d)$,

$$
\frac{1}{l} \partial_{x} \Phi(d, t)+\frac{\bar{E}}{\varphi_{0}^{2}} \Phi(d, t)=0
$$

determines the normal modes $k_{n}$ via the condition

$$
\left(k_{n} d\right) \tan \left(k_{n} d\right)=l d \frac{\bar{E}}{\varphi_{0}^{2}} .
$$

Using Eqs. (A14) and (A15) in Eq. (A12), we get

$$
\begin{aligned}
L_{0}= & \frac{1}{2} \sum_{n=1}^{\infty}\left\{c_{n} \dot{\phi}_{n}^{2}-l_{n}^{-1} \phi_{n}^{2}\right\}+\lambda g(t) \sum_{n=1}^{\infty} M_{n}^{(1)} \phi_{n}(t) \\
& +\lambda g(t) \tan \bar{\varphi} \sum_{n, m} M_{n m}^{(2)} \phi_{n}(t) \phi_{m}(t)
\end{aligned}
$$

with

$$
\begin{aligned}
& c_{n}=\frac{c d}{2}\left(1+\frac{\sin \left(2 k_{n} d\right)}{2 k_{n} d}\right), \\
& l_{n}^{-1}=\frac{\left(k_{n} d\right)^{2}}{2 l d}\left(1-\frac{\sin \left(2 k_{n} d\right)}{2 k_{n} d}\right)
\end{aligned}
$$

and

$$
\begin{aligned}
M_{n}^{(1)} & =(\delta \bar{\alpha}) \frac{\bar{E}}{\varphi_{0}} \cos \left(k_{n} d\right) \\
M_{n}^{(2)} & =\frac{1}{2} \bar{E} \varphi_{0}^{-2} \cos \left(k_{n} d\right) \cos \left(k_{m} d\right) .
\end{aligned}
$$

\section{b. Nonlinear terms and the Hamiltonian}

Using the normal mode decomposition [Eqs. (A14) and (A15)], we can rewrite Eq. (A13) as

$$
\begin{aligned}
L_{1}= & \lambda g(t) \sum_{n, m, l} M_{n m l}^{(3)} \phi_{n} \phi_{m} \phi_{l}+[1-\lambda \tan (\bar{\varphi}) g(t)] \\
& \times \sum_{n, m, l, p} M_{n m l p}^{(4)} \phi_{n} \phi_{m} \phi_{l} \phi_{p}
\end{aligned}
$$

with the coefficients

$$
\begin{aligned}
M_{n m l}^{(3)} & =\frac{1}{6} \bar{E} \varphi_{0}^{-3}(\delta \bar{\alpha}) \cos \left(k_{n} d\right) \cos \left(k_{m} d\right) \cos \left(k_{l} d\right), \\
M_{n m l p}^{(4)} & =\frac{1}{24} \bar{E} \varphi_{0}^{-4} \cos \left(k_{n} d\right) \cos \left(k_{m} d\right) \cos \left(k_{l} d\right) \cos \left(k_{p} d\right) .
\end{aligned}
$$

From Eqs. (A16) and (A17), we derive the Hamiltonian by means of a Legendre transformation and by introducing the conjugate variables $q_{n}=\partial L / \partial \dot{\phi}_{n}=c_{n} \dot{\phi}_{n} \quad$ with $L=L_{0}+L_{1}$ :

$$
\begin{aligned}
H= & \frac{1}{2} \sum_{n=1}^{\infty}\left\{c_{n}^{-1} q_{n}^{2}+l_{n}^{-1} \phi_{n}^{2}\right\}-\lambda g(t) \sum_{n=1}^{\infty} M_{n}^{(1)} \phi_{n} \\
& -\lambda g(t) \tan \bar{\varphi} \sum_{n, m} M_{n m}^{(2)} \phi_{n} \phi_{m}-\lambda g(t) \sum_{n, m, l} M_{n m l}^{(3)} \phi_{n} \phi_{m} \phi_{l} \\
& -[1-\lambda \tan (\bar{\varphi}) g(t)] \sum_{n, m, l, p} M_{n m l p}^{(4)} \phi_{n} \phi_{m} \phi_{l} \phi_{p} .
\end{aligned}
$$

Finally, we arrive to the quantum Hamiltonian by promoting the conjugate fields $\phi_{n}$ and $q_{n}$ to operators satisfying the bosonic commutation relation $\left[\phi_{n}, q_{m}\right]=i \delta_{n m}$.

Our next step is to rewrite the Hamiltonian in terms of the normal modes' creation and annihilation operators $\phi_{n}=$ $\kappa_{n}\left(a_{n}^{\dagger}+a_{n}\right)$ and $q_{n}=i \theta_{n}\left(a_{n}^{\dagger}-a_{n}\right)$, with $\kappa_{n}=\sqrt{1 / 2 \omega_{n} c_{n}}$ and $\theta_{n}=\sqrt{\omega_{n} c_{n} / 2}$. In this way, we obtain our desired result

$$
\begin{aligned}
H= & \sum_{n=1}^{\infty} \omega_{n} a_{n}^{\dagger} a_{n}-\lambda g(t) \sum_{n=1}^{\infty} \bar{M}_{n}^{(1)}\left(a_{n}^{\dagger}+a_{n}\right) \\
& -\lambda g(t) \tan \bar{\varphi} \sum_{n, m} \bar{M}_{n m}^{(2)}\left(a_{n}^{\dagger}+a_{n}\right)\left(a_{m}^{\dagger}+a_{m}\right) \\
& -\lambda g(t) \sum_{n, m, l} \bar{M}_{n m l}^{(3)}\left(a_{n}^{\dagger}+a_{n}\right)\left(a_{m}^{\dagger}+a_{m}\right)\left(a_{l}^{\dagger}+a_{l}\right) \\
& -[1-\lambda \tan (\bar{\varphi}) g(t)] \sum_{n, m, l, p} \bar{M}_{n m l p}^{(4)}\left(a_{n}^{\dagger}+a_{n}\right) \\
& \times\left(a_{m}^{\dagger}+a_{m}\right)\left(a_{l}^{\dagger}+a_{l}\right)\left(a_{p}^{\dagger}+a_{p}\right),
\end{aligned}
$$

with $\bar{M}_{n}^{(1)}=\kappa_{n} M_{n}^{(1)}, \bar{M}_{n m}^{(2)}=\kappa_{n} \kappa_{m} M_{n}^{(2)}, \bar{M}_{n m l}^{(3)}=\kappa_{n} \kappa_{m} \kappa_{l} M_{n m l}^{(3)}$, and $\bar{M}_{n m l p}^{(4)}=\kappa_{n} \kappa_{m} \kappa_{l} \kappa_{p} M_{n m l p}^{(4)}$.

\section{Three-photon processes}

Now we want to select certain third-order processes by means of the external modulation $g(t)$.

\section{a. Single-mode spontaneous parametric down-conversion}

The Hamiltonian we aim to engineer is of the form

$$
H_{1 \mathrm{M}} \sim a^{\dagger 3}+a^{3} \text {. }
$$

Restricting to a single normal mode $a \equiv a_{1}$, in the interaction picture with respect to $\omega a^{\dagger} a$, the Hamiltonian (A18) is 


$$
\begin{aligned}
H_{I}(t)= & -\lambda g(t) \bar{M}^{(1)}\left[a^{\dagger}(t)+a(t)\right]-\lambda g(t) \bar{M}^{(3)}\left[a^{\dagger}(t)\right. \\
& +a(t)]^{3}-[1-\lambda \tan (\bar{\varphi}) g(t)] \bar{M}^{(4)}\left[a^{\dagger}(t)+a(t)\right]^{4},
\end{aligned}
$$

with $a(t)=a \exp (-i \omega t)$. In Hamiltonian (A19), we have terms rotating with $\pm 3 \omega$. We choose the driving $g(t)$ to be of the form

$$
g(t)=\cos (3 \omega t)
$$

Upon this choice, we are left with the following nonrotating terms:

$$
H_{1 \mathrm{M}} \simeq-\lambda \bar{M}^{(3)}\left(a^{\dagger 3}+a^{3}\right),
$$

which is the single-mode interaction Hamiltonian from the main text. The total effective Hamiltonian in the rotatingwave approximation (RWA) is

$$
H_{\text {eff }}=-6 \bar{M}^{(4)} a^{\dagger 2} a^{2}-\lambda \bar{M}^{(3)}\left(a^{\dagger 3}+a^{3}\right),
$$

where the Kerr interaction is due to the static part of the SQUID potential.

\section{b. Three-mode spontaneous parametric down-conversion}

The Hamiltonian we wish to engineer is

$$
H_{3 \mathrm{M}} \sim a_{1} a_{2} a_{3}+a_{1}^{\dagger} a_{2}^{\dagger} a_{3}^{\dagger} .
$$

In the latter, we have terms rotating with $\pm\left(\omega_{1}+\omega_{2}+\omega_{3}\right)$. Therefore, we choose the driving $g(t)$ to be of the form

$$
g(t)=\cos \left[\left(\omega_{1}+\omega_{2}+\omega_{3}\right) t\right] .
$$

Upon this choice, we are left with the following nonrotating terms:

$$
H_{3 \mathrm{M}} \simeq-\lambda \bar{M}_{123}^{(3)}\left(a_{1} a_{2} a_{3}+a_{1}^{\dagger} a_{2}^{\dagger} a_{3}^{\dagger}\right),
$$

which is the three-mode interaction Hamiltonian from the main text. The total effective Hamiltonian in the RWA is

$$
\begin{aligned}
H_{\text {eff }}= & -6 \sum_{i} \bar{M}_{i}^{(4)} a_{i}^{\dagger 2} a_{i}^{2}-4 \sum_{i j} \bar{M}_{i j}^{(4)}\left(a_{i}^{\dagger} a_{i}\right)\left(a_{j}^{\dagger} a_{j}\right) \\
& -\lambda \bar{M}_{123}^{(3)}\left(a_{1} a_{2} a_{3}+a_{1}^{\dagger} a_{2}^{\dagger} a_{3}^{\dagger}\right) .
\end{aligned}
$$

Similar to the previous case, the Kerr interactions are due to the static part of the SQUID potential.

\section{c. Effects of the Kerr interactions}

Similar to the case of standard two-photon SPDC, the Kerr terms in Eqs. (21) and (24) help to stabilize the cavity amplitude under steady-state pumping conditions through an autonomous feedback mechanism [54]. That is, as the cavity amplitude increases, the Kerr terms induce frequency shifts that move the modes out of resonance with the pump. At higher powers, we also expect that the Kerr terms may limit the degree of third-order correlation that can be achieved, in much the same that they limit conventional squeezing in two-photon SPDC.

\section{APPENDIX B: THIRD-ORDER CORRELATION ANALYSIS OF TRISQUEEZED STATES}

\section{Three-mode SPDC}

In this section, we analyze the predicted transformation properties of the correlators considered in the paper. As a first example, we take the three-mode case governed by $\mathcal{H}_{3 \mathrm{M}}$ as an example, analyzing the skewness of the generalized quadrature $I_{\phi \theta}=I_{1} \cos (\phi) \cos (\theta)-I_{2} \sin (\phi)+$ $I_{3} \cos (\phi) \sin (\theta)$. As in the text, we use the convention $I_{k}=a_{k}+a_{k}^{\dagger}$.

To predict the form of $\left\langle I_{\phi \theta}^{3}\right\rangle$, we consider our system evolving from the vacuum state under the action of

$$
\mathcal{H}_{3 \mathrm{M}} \simeq-\lambda \bar{M}_{123}^{(3)}\left(a_{1} a_{2} a_{3} e^{i \eta}+a_{1}^{\dagger} a_{2}^{\dagger} a_{3}^{\dagger} e^{-i \eta}\right),
$$

where we slightly generalize Eq. (A23) to include the pump phase $\eta$. We then note that we can estimate the expectation value of any observable $\mathcal{O}$ using perturbation theory as

$$
\langle\mathcal{O}\rangle=\langle 0|\mathcal{O}| 0\rangle-i t\left\langle 0\left|\mathcal{O H}_{3 \mathrm{M}}\right| 0\right\rangle+i t\left\langle 0\left|\mathcal{H}_{3 \mathrm{M}} \mathcal{O}\right| 0\right\rangle+O\left(\lambda^{2}\right) .
$$

Now expanding $I_{\phi \theta}^{3}$ and noting that quadrature operators of different modes commute, the skewness is

$$
\begin{aligned}
\left\langle I_{\phi \theta}^{3}\right\rangle= & \left\langle\left[\cos (\phi) \cos (\theta) I_{1}-\sin (\phi) I_{2}+\cos (\phi) \sin (\theta) I_{3}\right]^{3}\right\rangle \\
= & 1\left\langle I_{1} I_{1} I_{1}\right\rangle \cos ^{3}(\phi) \cos ^{3}(\theta)-1\left\langle I_{2} I_{2} I_{2}\right\rangle \sin ^{3}(\phi)+1\left\langle I_{3} I_{3} I_{3}\right\rangle \cos ^{3}(\phi) \sin ^{3}(\theta)-3\left\langle I_{1} I_{1} I_{2}\right\rangle \cos ^{2}(\phi) \cos ^{2}(\theta) \sin (\phi) \\
& +3\left\langle I_{1} I_{1} I_{3}\right\rangle \cos ^{3}(\phi) \cos ^{2}(\theta) \sin (\theta)+3\left\langle I_{1} I_{2} I_{2}\right\rangle \cos (\phi) \cos (\theta) \sin ^{2}(\phi)-6\left\langle I_{1} I_{2} I_{3}\right\rangle \cos ^{2}(\phi) \cos (\theta) \sin (\phi) \sin (\theta) \\
& +3\left\langle I_{1} I_{3} I_{3}\right\rangle \cos ^{3}(\phi) \cos (\theta) \sin ^{2}(\theta)+3\left\langle I_{2} I_{2} I_{3}\right\rangle \sin ^{2}(\phi) \cos (\phi) \sin (\theta)-3\left\langle I_{2} I_{3} I_{3}\right\rangle \sin (\phi) \cos ^{2}(\phi) \sin ^{2}(\theta) .
\end{aligned}
$$

Importantly, we note that each correlator contributes a different geometric form factor to $\left\langle I_{\phi \theta}^{3}\right\rangle$ allowing us to identify the correlations present from the shape of $\left\langle I_{\phi \theta}^{3}\right\rangle$. 
To find an explicit form generated by our Hamiltonian, we observe that

$$
\left\langle 0\left|I_{\phi \theta}^{3}\right| 0\right\rangle=0,
$$

as the vacuum is a Gaussian state. In $\left\langle 0\left|I_{\phi \theta}^{3} \mathcal{H}_{3 M}\right| 0\right\rangle$ and $\left\langle 0\left|\mathcal{H}_{3 \mathrm{M}} I_{\phi \theta}^{3}\right| 0\right\rangle$, the only nonzero expectation values come from the quadrature products containing all three modes, i.e., $I_{1} I_{2} I_{3}$. In particular,

$$
\begin{aligned}
\left\langle 0\left|I_{\phi \theta}^{3} \mathcal{H}_{3 \mathrm{M}}\right| 0\right\rangle= & 6 \lambda \bar{M}_{123}^{(3)} \cos ^{2}(\phi) \cos (\theta) \\
& \times \sin (\phi) \sin (\theta) e^{-i \eta} \overbrace{\left\langle 0\left|a_{1} a_{2} a_{3} a_{1}^{\dagger} a_{2}^{\dagger} a_{3}^{\dagger}\right| 0\right\rangle}^{=1}, \\
\left\langle 0\left|\mathcal{H}_{3 \mathrm{M}} I_{\phi \theta}^{3}\right| 0\right\rangle= & 6 \lambda \bar{M}_{123}^{(3)} \cos ^{2}(\phi) \cos (\theta) \\
& \times \sin (\phi) \sin (\theta) e^{i \eta} \overbrace{\left\langle 0\left|a_{1} a_{2} a_{3} a_{1}^{\dagger} a_{2}^{\dagger} a_{3}^{\dagger}\right| 0\right\rangle}^{=1} .
\end{aligned}
$$

Putting the above together, we find

$$
\begin{aligned}
\left\langle I_{\phi \theta}^{3}\right\rangle & \approx i t\left(\left\langle 0\left|\mathcal{H}_{3 \mathrm{M}} I_{\phi \theta}^{3}\right| 0\right\rangle-\left\langle 0\left|I_{\phi \theta}^{3} \mathcal{H}_{3 \mathrm{M}}\right| 0\right\rangle\right) \\
& =-12 \lambda t \bar{M}_{123}^{(3)} \cos ^{2}(\phi) \cos (\theta) \sin (\phi) \sin (\theta) \sin (\eta) .
\end{aligned}
$$

Similarly, we can compute $\left\langle Q_{\phi \theta}^{3}\right\rangle$ where $I_{k}$ is now replaced by $Q_{k}=-i\left(a_{k}-a_{k}^{\dagger}\right)$. With the same approach, we obtain

$$
\left\langle Q_{\phi \theta}^{3}\right\rangle \approx-12 \lambda t \bar{M}_{123}^{(3)} \cos ^{2}(\phi) \cos (\theta) \sin (\phi) \sin (\theta) \cos (\eta) .
$$

Regarding the role of the pump phase $\eta$, we observe that the choice of $\eta$ simply rotates correlations between the $I$ and $Q$ quadratures. The normalized version of Eq. (B1) with $\eta=\pi / 2$ gives the theory plot in Fig. 5 of the main text. As this particular form factor is contributed only by the three-mode coskewness $\left\langle I_{1} I_{2} I_{3}\right\rangle$, this shape is a distinctive fingerprint of $\mathcal{H}_{3 \mathrm{M}}$. Its match with the experimental data is therefore a strong indication of the purity of the downconversion process.

\section{Two-mode SPDC}

For two-mode SPDC, we consider the Hamiltonian

$$
\mathcal{H}_{2 \mathrm{M}} \simeq-\lambda \bar{M}_{112}^{(3)}\left(a_{1} a_{1} a_{2} e^{i \eta}+a_{1}^{\dagger} a_{1}^{\dagger} a_{2}^{\dagger} e^{-i \eta}\right) .
$$

Following the main text, we consider the skewness of the generalized two-mode quadrature $I_{\phi \theta}=I_{1} \cos (\phi) \cos (\theta)-$ $Q_{1} \sin (\phi)+I_{2} \cos (\phi) \sin (\theta)$. With a similar expansion as above, we observe that, under the action of $\mathcal{H}_{2 \mathrm{M}}$, the only nonzero skewness terms are those involving mode 1 twice and mode 2 once, i.e., $\left\langle I_{1} I_{1} I_{2}\right\rangle,\left\langle I_{1} Q_{1} I_{2}\right\rangle$, and $\left\langle Q_{1} Q_{1} I_{2}\right\rangle$. Accordingly, we find

$$
\begin{aligned}
\left\langle I_{\phi \theta}^{3}\right\rangle \approx & i t\left(\left\langle 0\left|\mathcal{H}_{2 \mathrm{M}} I_{\varphi}^{3}\right| 0\right\rangle-\left\langle 0\left|I_{\varphi}^{3} \mathcal{H}_{2 \mathrm{M}}\right| 0\right\rangle\right) \\
= & 12 \lambda \bar{M}_{112}^{(3)} t\left[\cos ^{3}(\phi) \cos ^{2}(\theta) \sin (\theta)\right. \\
& \left.-\sin ^{2}(\phi) \cos (\phi) \sin (\theta)\right] \sin (\eta) \\
& -24 \lambda \bar{M}_{112}^{(3)} t\left[\cos ^{2}(\phi) \cos (\theta) \sin (\phi) \sin (\theta)\right] \cos (\eta)
\end{aligned}
$$

The terms proportional to $\sin (\eta)$ are contributed by $\left\langle I_{1} I_{1} I_{2}\right\rangle$ and $\left\langle Q_{1} Q_{1} I_{2}\right\rangle$, while the term proportional to $\cos (\eta)$ is contributed by $\left\langle I_{1} Q_{1} I_{2}\right\rangle$. A slight offset from $\eta=\pi / 2$ therefore explains the residual value of $\left\langle I_{1} Q_{1} I_{2}\right\rangle$ in Fig. 4 of the main text. For the theory plot in Fig. 5(b), we use exactly $\eta=\pi / 2$.

\section{Single-mode SPDC}

With the same approach, we can also predict the skewness of the generalized quadrature $I_{\varphi}=I \cos (\varphi)+$ $Q \sin (\varphi)$, for different $\varphi$ under the interaction given by $\mathcal{H}_{1 M}$ [see Eq. (A20)]. Using our definition of $I$ and $Q$, we can expand $I_{\varphi}^{3}$ as

$$
\begin{aligned}
I_{\varphi}^{3}= & a^{3} e^{-i 3 \varphi}+a^{\dagger 3} e^{i 3 \varphi}+a^{2} a^{\dagger} e^{-i \varphi}+a a^{\dagger} a e^{-i \varphi}+a a^{\dagger} a^{\dagger} e^{i \varphi} \\
& +a^{\dagger} a a e^{-i \varphi}+a^{\dagger} a a^{\dagger} e^{i \varphi}+a^{\dagger} a^{\dagger} a e^{i \varphi} .
\end{aligned}
$$

Following the same approach as above, we find

$$
\begin{aligned}
\left\langle 0\left|\mathcal{H}_{1 \mathrm{M}} I_{\varphi}^{3}\right| 0\right\rangle & =-\lambda \bar{M}^{(3)}\left\langle 0\left|a^{3} e^{i \eta} e^{i 3 \varphi} a^{\dagger 3}\right| 0\right\rangle \\
& =-6 \lambda \bar{M}^{(3)} e^{i(\eta+3 \varphi)}
\end{aligned}
$$

and

$$
\begin{aligned}
\left\langle 0\left|I_{\varphi}^{3} \mathcal{H}_{1 \mathrm{M}}\right| 0\right\rangle & =-\lambda \bar{M}^{(3)}\left\langle 0\left|a^{3} e^{-i \eta} e^{-i 3 \varphi} a^{\dagger 3}\right| 0\right\rangle \\
& =-6 \lambda \bar{M}^{(3)} e^{-i(\eta+3 \varphi)},
\end{aligned}
$$

finally giving

$$
\begin{aligned}
\left\langle I_{\varphi}^{3}\right\rangle & \approx i t\left(\left\langle 0\left|\mathcal{H}_{1 \mathrm{M}} I_{\varphi}^{3}\right| 0\right\rangle-\left\langle 0\left|I_{\varphi}^{3} \mathcal{H}_{1 \mathrm{M}}\right| 0\right\rangle\right) \\
& =12 t \lambda \bar{M}^{(3)} \sin (3 \varphi+\eta) .
\end{aligned}
$$

[1] S. Friberg, C. K. Hong, and L. Mandel, Measurement of Time Delays in the Parametric Production of Photon Pairs, Phys. Rev. Lett. 54, 2011 (1985).

[2] W. Tittel, J. Brendel, H. Zbinden, and N. Gisin, Violation of Bell Inequalities by Photons More Than $10 \mathrm{~km}$ Apart, Phys. Rev. Lett. 81, 3563 (1998). 
[3] D. C. Burnham and D. L. Weinberg, Observation of Simultaneity in Parametric Production of Optical Photon Pairs, Phys. Rev. Lett. 25, 84 (1970).

[4] W. Tittel, J. Brendel, H. Zbinden, and N. Gisin, Quantum Cryptography Using Entangled Photons in Energy-Time Bell States, Phys. Rev. Lett. 84, 4737 (2000).

[5] P. Walther, K. J. Resch, T. Rudolph, E. Schenck, H. Weinfurter, V. Vedral, M. Aspelmeyer, and A. Zeilinger, Experimental One-Way Quantum Computing, Nature (London) 434, 169 (2005).

[6] N. Didier, A. Kamal, W. D. Oliver, A. Blais, and A. A. Clerk, Heisenberg-Limited Qubit Read-Out with Two-Mode Squeezed Light, Phys. Rev. Lett. 115, 093604 (2015).

[7] T. Yamamoto, K. Inomata, M. Watanabe, K. Matsuba, T. Miyazaki, W. D. Oliver, Y. Nakamura, and J. S. Tsai, FluxDriven Josephson Parametric Amplifier, Appl. Phys. Lett. 93, 042510 (2008).

[8] B. Yurke, P. G. Kaminsky, R. E. Miller, E. A. Whittaker, A. D. Smith, A. H. Silver, and R. W. Simon, Observation of 4.2-K Equilibrium-Noise Squeezing via a JosephsonParametric Amplifier, Phys. Rev. Lett. 60, 764 (1988).

[9] R. Movshovich, B. Yurke, P. G. Kaminsky, A. D. Smith, A. H. Silver, R. W. Simon, and M. V. Schneider, Observation of Zero-Point Noise Squeezing via a Josephson-Parametric Amplifier, Phys. Rev. Lett. 65, 1419 (1990).

[10] M. Cooper, L. J. Wright, C. Söller, and B. J. Smith, Experimental Generation of Multi-Photon Fock States, Opt. Express 21, 5309 (2013).

[11] P. G. Kwiat, K. Mattle, H. Weinfurter, A. Zeilinger, A. V. Sergienko, and Y. Shih, New High-Intensity Source of Polarization-Entangled Photon Pairs, Phys. Rev. Lett. 75, 4337 (1995).

[12] C. M. Caves and B. L. Schumaker, New Formalism for Two-Photon Quantum Optics. I. Quadrature Phases and Squeezed States, Phys. Rev. A 31, 3068 (1985).

[13] B. L. Schumaker and C. M. Caves, New Formalism for Two-Photon Quantum Optics. II. Mathematical Foundation and Compact Notation, Phys. Rev. A 31, 3093 (1985).

[14] R. A. Fisher, M. M. Nieto, and V. D. Sandberg, Impossibility of Naively Generalizing Squeezed Coherent States, Phys. Rev. D 29, 1107 (1984).

[15] M. Hillery, M. S. Zubairy, and K. Wódkiewicz, Squeezing in Higher Order Nonlinear Optical Processes, Phys. Lett. A 103, 259 (1984).

[16] S. L. Braunstein and R. I. McLachlan, Generalized Squeezing, Phys. Rev. A 35, 1659 (1987).

[17] S. L. Braunstein and C. M. Caves, Phase and Homodyne Statistics of Generalized Squeezed States, Phys. Rev. A 42, 4115 (1990).

[18] M. Hillery, Photon Number Divergence in the Quantum Theory of $n$-Photon Down Conversion, Phys. Rev. A 42, 498 (1990).

[19] K. Banaszek and P. L. Knight, Quantum Interference in Three-Photon Down-Conversion, Phys. Rev. A 55, 2368 (1997).

[20] T. Felbinger, S. Schiller, and J. Mlynek, Oscillation and Generation of Nonclassical States in Three-Photon DownConversion, Phys. Rev. Lett. 80, 492 (1998).
[21] M. K. Olsen, L. I. Plimak, and M. Fleischhauer, QuantumTheoretical Treatments of Three-Photon Processes, Phys. Rev. A 65, 053806 (2002).

[22] K. Marshall, R. Pooser, G. Siopsis, and C. Weedbrook, Repeat-until-Success Cubic Phase Gate for Universal Continuous-Variable Quantum Computation, Phys. Rev. A 91, 032321 (2015).

[23] F. Arzani, N. Treps, and G. Ferrini, Polynomial Approximation of Non-Gaussian Unitaries by Counting One Photon at a Time, Phys. Rev. A 95, 052352 (2017).

[24] K. Bencheikh, F. Gravier, J. Douady, A. Levenson, and B. Boulanger, Triple Photons: A Challenge in Nonlinear and Quantum Optics, C.R. Phys. 8, 206 (2007).

[25] E. A. R. González, A. Borne, B. Boulanger, J. A. Levenson, and K. Bencheikh, Continuous-Variable Triple-Photon States Quantum Entanglement, Phys. Rev. Lett. 120, 043601 (2018).

[26] N. A. Borshchevskaya, K. G. Katamadze, S. P. Kulik, and M. V. Fedorov, Three-Photon Generation by Means of Third-Order Spontaneous Parametric Down-Conversion in Bulk Crystals, Laser Phys. Lett. 12, 115404 (2015).

[27] M. Corona, K. Garay-Palmett, and A. B. U'Ren, Experimental Proposal for the Generation of Entangled Photon Triplets by Third-Order Spontaneous Parametric Downconversion in Optical Fibers, Opt. Lett. 36, 190 (2011).

[28] M. Akbari and A. A. Kalachev, Third-Order Spontaneous Parametric Down-Conversion in a Ring Microcavity, Laser Phys. Lett. 13, 115204 (2016).

[29] A. Cavanna, F. Just, X. Jiang, G. Leuchs, M. V. Chekhova, P. S. J. Russell, and N. Y. Joly, Hybrid PhotonicCrystal Fiber for Single-Mode Phase Matched Generation of Third Harmonic and Photon Triplets, Optica 3, 952 (2016).

[30] K. Zelaya, S. Dey, and V. Hussin, Generalized Squeezed States, Phys. Lett. A 382, 3369 (2018).

[31] S. Ding, G. Maslennikov, R. Hablützel, and D. Matsukevich, Quantum Simulation with a Trilinear Hamiltonian, Phys. Rev. Lett. 121, 130502 (2018).

[32] S. Ghose and B. C. Sanders, Non-Gaussian States of Light as an Offline Resource for Universal Continuous Variable Quantum Information Processing, in Quantum Communications and Quantum Imaging III, Vol. 5893, edited by R. E. Meyers and Y. Shih (International Society for Optics and Photonics, Bellingham, WA, 2005), p. 58930X.

[33] M. Gu, C. Weedbrook, N. C. Menicucci, T. C. Ralph, and P. van Loock, Quantum Computing with ContinuousVariable Clusters, Phys. Rev. A 79, 062318 (2009).

[34] R. Takagi and Q. Zhuang, Convex Resource Theory of NonGaussianity, Phys. Rev. A 97, 062337 (2018).

[35] F. Albarelli, M. G. Genoni, M. G. A. Paris, and A. Ferraro, Resource Theory of Quantum Non-Gaussianity and Wigner Negativity, Phys. Rev. A 98, 052350 (2018).

[36] D. Gottesman, A. Kitaev, and J. Preskill, Encoding a Qubit in an Oscillator, Phys. Rev. A 64, 012310 (2001).

[37] S. Agne, T. Kauten, J. Jin, E. Meyer-Scott, J. Z. Salvail, D. R. Hamel, K. J. Resch, G. Weihs, and T. Jennewein, Observation of Genuine Three-Photon Interference, Phys. Rev. Lett. 118, 153602 (2017). 
[38] D. R. Hamel, L. K. Shalm, H. Hübel, A. J. Miller, F. Marsili, V. B. Verma, R. P. Mirin, S. W. Nam, K. J. Resch, and T. Jennewein, Direct Generation of Three-Photon Polarization Entanglement, Nat. Photonics 8, 801 (2014).

[39] M. Hillery, V. Bužek, and A. Berthiaume, Quantum Secret Sharing, Phys. Rev. A 59, 1829 (1999).

[40] E. Flurin, N. Roch, J. D. Pillet, F. Mallet, and B. Huard, Superconducting Quantum Node for Entanglement and Storage of Microwave Radiation, Phys. Rev. Lett. 114, 090503 (2015).

[41] C. W. S. Chang, M. Simoen, J. Aumentado, C. Sabín, P. Forn-Díaz, A. M. Vadiraj, F. Quijandría, G. Johansson, I. Fuentes, and C. M. Wilson, Generating Multimode Entangled Microwaves with a Superconducting Parametric Cavity, Phys. Rev. Applied 10, 044019 (2018).

[42] J. Douady and B. Boulanger, Experimental Demonstration of a Pure Third-Order Optical Parametric Downconversion Process, Opt. Lett. 29, 2794 (2004).

[43] M. Khoshnegar, T. Huber, A. Predojević, D. Dalacu, M. Prilmüller, J. Lapointe, X. Wu, P. Tamarat, B. Lounis, P. Poole, G. Weihs, and H. Majedi, A Solid State Source of Photon Triplets Based on Quantum Dot Molecules, Nat. Commun. 8, 15716 (2017).

[44] I.-M. Svensson, A. Bengtsson, P. Krantz, J. Bylander, V. Shumeiko, and P. Delsing, Period-Tripling Subharmonic Oscillations in a Driven Superconducting Resonator, Phys. Rev. B 96, 174503 (2017).

[45] N. Lörch, Y. Zhang, C. Bruder, and M. I. Dykman, Quantum State Preparation for Coupled Period Tripling Oscillators, Phys. Rev. Research 1, 023023 (2019).

[46] C. Eichler, D. Bozyigit, C. Lang, M. Baur, L. Steffen, J. M. Fink, S. Filipp, and A. Wallraff, Observation of Two-Mode
Squeezing in the Microwave Frequency Domain, Phys. Rev. Lett. 107, 113601 (2011).

[47] E. Flurin, N. Roch, F. Mallet, M. H. Devoret, and B. Huard, Generating Entangled Microwave Radiation over Two Transmission Lines, Phys. Rev. Lett. 109, 183901 (2012).

[48] E. Zakka-Bajjani, F. Nguyen, M. Lee, L. R. Vale, R. W. Simmonds, and J. Aumentado, Quantum Superposition of a Single Microwave Photon in Two Different 'Colour' States, Nat. Phys. 7, 599 (2011).

[49] M. Simoen, C. W. S. Chang, P. Krantz, J. Bylander, W. Wustmann, V. Shumeiko, P. Delsing, and C. M. Wilson, Characterization of a Multimode Coplanar Waveguide Parametric Amplifier, J. Appl. Phys. 118, 154501 (2015).

[50] J. R. Johansson, G. Johansson, C. M. Wilson, and F. Nori, Dynamical Casimir Effect in Superconducting Microwave Circuits, Phys. Rev. A 82, 052509 (2010).

[51] Z. Leghtas, S. Touzard, I. M. Pop, A. Kou, B. Vlastakis, A. Petrenko, K. M. Sliwa, A. Narla, S. Shankar, M. J. Hatridge, M. Reagor, L. Frunzio, R. J. Schoelkopf, M. Mirrahimi, and M. H. Devoret, Confining the State of Light to a Quantum Manifold by Engineered Two-Photon Loss, Science 347, 853 (2015).

[52] R. Simon, N. Mukunda, and B. Dutta, Quantum-Noise Matrix for Multimode Systems: U(n) Invariance, Squeezing, and Normal Forms, Phys. Rev. A 49, 1567 (1994).

[53] C. Eichler and A. Wallraff, Controlling the Dynamic Range of a Josephson Parametric Amplifier, EPJ Quantum Technol. 1, 2 (2014).

[54] C. M. Wilson, T. Duty, M. Sandberg, F. Persson, V. Shumeiko, and P. Delsing, Photon Generation in an Electromagnetic Cavity with a Time-Dependent Boundary, Phys. Rev. Lett. 105, 233907 (2010). 